Washington University School of Medicine Digital Commons@Becker

Open Access Publications

2021

\title{
Targeting primary and metastatic uveal melanoma with a G protein inhibitor
}

\author{
Michael D. Onken \\ Washington University School of Medicine in St. Louis \\ Carol M. Makepeace \\ Washington University School of Medicine in St. Louis \\ Kevin M. Kaltenbronn \\ Washington University School of Medicine in St. Louis \\ Joelle Choi \\ Washington University School of Medicine in St. Louis \\ Leonel Hernandez-Aya \\ Washington University School of Medicine in St. Louis
}

See next page for additional authors

Follow this and additional works at: https://digitalcommons.wustl.edu/open_access_pubs

Please let us know how this document benefits you.

\author{
Recommended Citation \\ Onken, Michael D.; Makepeace, Carol M.; Kaltenbronn, Kevin M.; Choi, Joelle; Hernandez-Aya, Leonel; \\ Weilbaecher, Katherine N.; Piggott, Kisha D.; Rao, P. Kumar; Yuede, Carla M.; Dixon, Alethia J.; Osei-Owusu, \\ Patrick; Cooper, John A.; and Blumer, Kendall J., "Targeting primary and metastatic uveal melanoma with \\ a G protein inhibitor." Journal of Biological Chemistry. 296, 100403 (2021). \\ https://digitalcommons.wustl.edu/open_access_pubs/10125
}

This Open Access Publication is brought to you for free and open access by Digital Commons@Becker. It has been accepted for inclusion in Open Access Publications by an authorized administrator of Digital Commons@Becker. For more information, please contact vanam@wustl.edu. 


\section{Authors}

Michael D. Onken, Carol M. Makepeace, Kevin M. Kaltenbronn, Joelle Choi, Leonel Hernandez-Aya, Katherine N. Weilbaecher, Kisha D. Piggott, P. Kumar Rao, Carla M. Yuede, Alethia J. Dixon, Patrick OseiOwusu, John A. Cooper, and Kendall J. Blumer 


\section{Targeting primary and metastatic uveal melanoma with a G protein inhibitor}

Received for publication, December 29, 2020, and in revised form, January 25, 2021 Published, Papers in Press, February 10, 2021, https://doi.org/10.1016/j.jbc.2021.100403

Michael D. Onken ${ }^{1}{ }^{\circledR}$, Carol M. Makepeace ${ }^{2}$, Kevin M. Kaltenbronn ${ }^{2}$, Joelle Choi ${ }^{2}$, Leonel Hernandez-Aya ${ }^{3}$, Katherine N. Weilbaecher ${ }^{3}$, Kisha D. Piggott ${ }^{4}$, P. Kumar Rao ${ }^{4}$, Carla M. Yuede ${ }^{5}$, Alethia J. Dixon ${ }^{6}$, Patrick Osei-Owusu ${ }^{6}$, John A. Cooper , and Kendall J. Blumer ${ }^{2, *} \mathbb{C}$ From the Departments of ${ }^{1}$ Biochemistry and Molecular Biophysics, ${ }^{2}$ Cell Biology and Physiology, ${ }^{3}$ Medicine, ${ }^{4}$ Ophthalmology and Visual Sciences, and ${ }^{5}$ Psychiatry, Washington University School of Medicine, St Louis, Missouri, USA; and ${ }^{6}$ Department of Pharmacology \& Physiology, Drexel University College of Medicine, Philadelphia, Pennsylvania, USA

Edited by Henrik Dohlman

Uveal melanoma (UM) is the most common intraocular tumor in adults. Nearly half of UM patients develop metastatic disease and often succumb within months because effective therapy is lacking. A novel therapeutic approach has been suggested by the discovery that UM cell lines driven by mutant constitutively active Gq or G11 can be targeted by FR900359 (FR) or YM-254890, which are bioavailable, selective inhibitors of the Gq/11/14 subfamily of heterotrimeric $G$ proteins. Here, we have addressed the therapeutic potential of FR for UM. We found that FR inhibited all oncogenic Gq/11 mutants reported in UM. FR arrested growth of all Gq/11-driven UM cell lines tested, but induced apoptosis only in a few. Similarly, FR inhibited growth of, but did not efficiently kill, UM tumor cells from biopsies of primary or metastatic tumors. FR evoked melanocytic redifferentiation of UM tumor cells with low (class 1), but not high (class 2), metastatic potential. FR administered systemically below its $L D_{50}$ strongly inhibited growth of PDX-derived class 1 and class 2 UM tumors in mouse xenograft models and reduced blood pressure transiently. FR did not regress xenografted UM tumors or significantly affect heart rate, liver function, hematopoiesis, or behavior. These results indicated the existence of a therapeutic window in which FR can be explored for treating UM and potentially other diseases caused by constitutively active Gq/11.

Mutant constitutively active forms of $\mathrm{Gq}$ or $\mathrm{G} 11$ (Gq/11) $\alpha$-subunits of heterotrimeric $G$ proteins cause uveal melanoma (UM) (1-3) and several other diseases and disorders (4-11). UM is particularly devastating because patients with metastatic disease often succumb within months due to lack of effective therapy (12). Clinical trials of metastatic UM have shown little benefit of cytotoxic chemotherapeutics, immune checkpoint inhibitors, or small-molecule inhibitors of oncogenic signaling proteins including protein kinase $C$ and MEK (13). New therapeutic strategies for metastatic UM clearly are warranted.

* For correspondence: Kendall J. Blumer, kblumer@wustl.edu.
Recent evidence suggests that mutant constitutively active Gq/11 could be targeted therapeutically in UM and other diseases (14). Oncogenic Gq/11 $\alpha$-subunits are constitutively active due to defects in hydrolyzing GTP to GDP, the ratelimiting step for $\mathrm{G}$ protein deactivation. Nevertheless, oncogenic Gq/11 can be inhibited by FR900359 (FR) or YM-254890 (YM) (15-20), a pair of closely related, bioavailable cyclic depsipeptides that allosterically inhibit GDP release by the Gq/ $11 / 14$ subfamily $(16,21)$. Inhibition is thought to occur because oncogenic Gq/11 $\alpha$-subunits can spontaneously release GTP, stochastically bind GDP, bind YM or FR (YM/FR) and Gßy subunits (17), forming complexes resistant to activation by $\mathrm{G}$-protein-coupled receptors $(14,16)$ and diminishing downstream oncogenic signaling (17-20). In response to YM/FR, UM cell lines driven by oncogenic Gq/11 arrest the cell cycle and can undergo apoptosis and/or redifferentiation into melanocytic-like cells (17-20). In contrast, BRAF-driven UM cell lines, which express wild-type Gq/11, are unaffected (17-20).

The therapeutic potential of YM/FR in UM is an important opportunity based on this evidence. Critical issues and questions remain to be addressed, however. YM/FR may exert deleterious as well as therapeutically beneficial effects, because they do not discriminate between wild-type and oncogenic Gq/11 (14), and target Gq/11-dependent physiological systems (21-24) essential for homeostasis and viability (25-28). Indeed, YM/FR administered systemically at levels that reduce $\mathrm{Gq} / 11$ activity in host systems greater than $50 \%$ is likely to be lethal, as suggested by gene dosage studies of $\mathrm{Gq} / 11$ knockout mice (29). Indeed, how strongly oncogenic $\mathrm{Gq} / 11$ signaling in UM tumors must be inhibited for therapeutic effect remains unknown. Furthermore, although recent studies have shown that YM/FR can inhibit growth of UM tumors in mouse xenograft models $(19,30)$, questions left unanswered are whether YM/FR can target primary and/or metastatic UM tumor cells from patients, whether YM/FR responsiveness is affected by tumor-intrinsic factors, including the type of oncogenic Gq/11 mutation, tumor class or metastatic potential, or whether systemic administration of YM/FR at levels sufficient to target UM tumor xenografts has serious 
deleterious effects on viability or host physiological systems. Here, we have addressed all of these important issues.

\section{Results}

FR inhibits all oncogenic $\mathrm{Gq}$ and $\mathrm{G} 11$ mutants that drive uveal melanoma

At least ten different constitutively active $\mathrm{Gq} / 11$ mutants have been identified in $\operatorname{UM}(2,31,32)$. Only four had been studied previously and shown to be inhibited by FR or YM $(17-20)$. To extend this analysis, we transiently expressed each of ten different Gq/11 mutants that drive UM (Fig. 1A) with a Gq/11-driven transcriptional reporter in a common cellular context (HEK293 cells) to exclude extrinsic factors that might affect the results. We found that each mutant $G$ protein induced reporter expression and was inhibited by $\mathrm{FR}$ with similar potency ( $\mathrm{IC}_{50} 1.9-3.8 \mathrm{nM}$ ) and efficacy (Fig. 1A).

In contrast, we found that four UM cell lines (Fig. 1B) driven by three different oncogenic $\mathrm{Gq} / 11$ mutants responded to $\mathrm{FR}$ in quantitatively or qualitatively different ways. FR inhibited growth of all four Gq/11-driven UM cell lines, but with potencies differing by as much as $\sim 80$-fold ( $\mathrm{IC}_{50} 0.5 \mathrm{nM}$ (MP41 cells), $38 \mathrm{nM}$ (Mel270 cells); Fig. 1B). Whereas FR arrested the cell cycle of all Gq/11-driven UM cell lines, as indicated by reduction of S- and G2/M-phase cells (Figs. $1 C$ and S1), it induced significant apoptosis only in two of them (92.1 and Mel270 cells), as indicated by accumulation of cells with sub-G0/G1 DNA content (Figs. $1 C$ and S1). FR did not affect proliferation or survival of a BRAF(V600E)-driven UM cell line (Fig. 1B), as shown

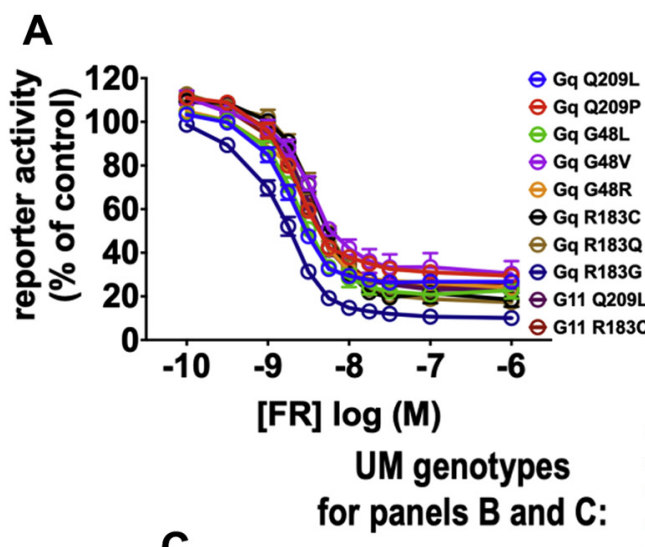

B

C
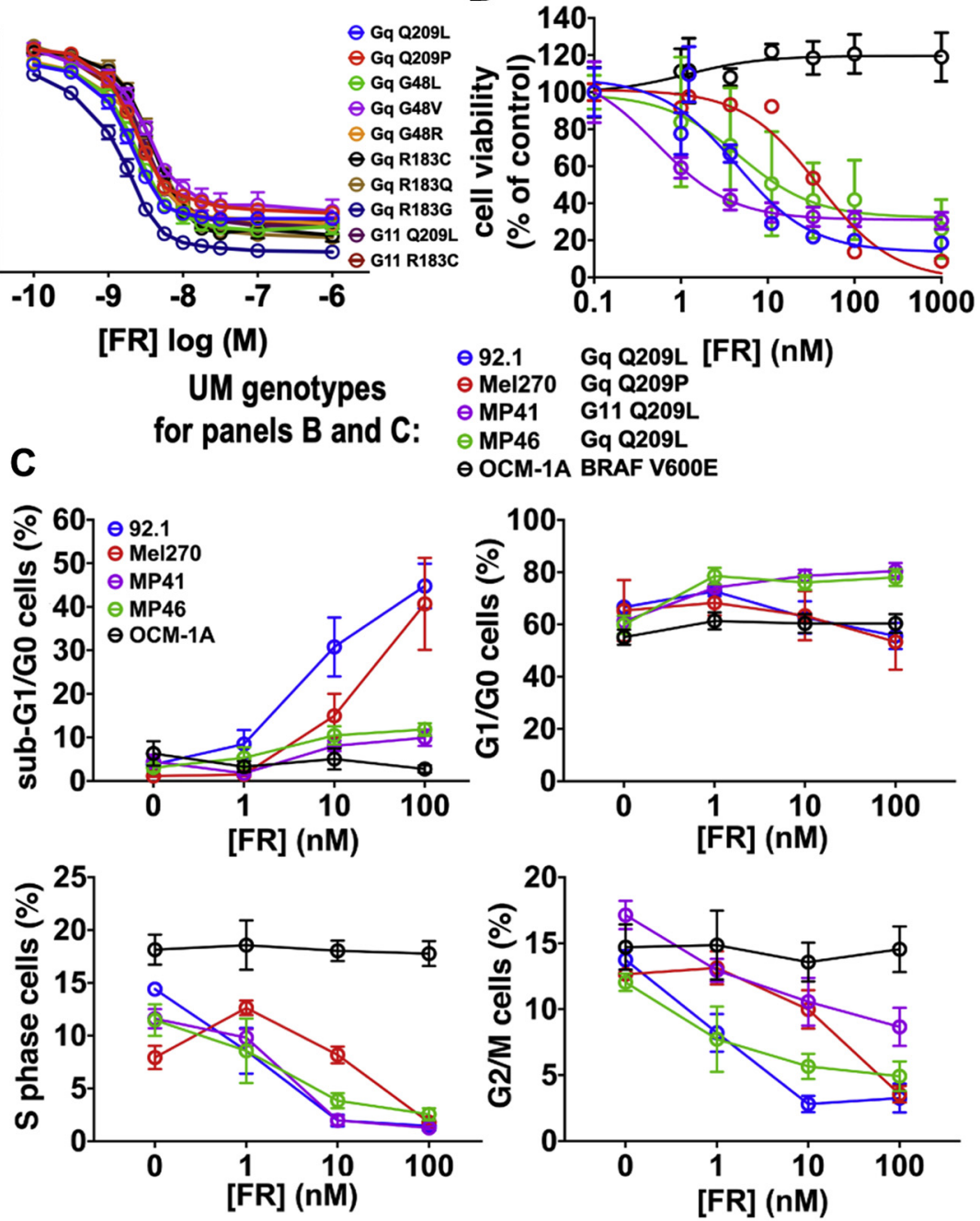

Figure 1. Effects of FR on constitutively active Gq/11 mutants and UM cell lines. $A$, effects of FR on expression of an SRE.L reporter driven by transfection of oncogenic Gq/11 mutants in HEK293 cells. FR inhibited all ten Gq/11 mutants reported in UM, with IC50s between $1.9 \mathrm{nM}$ (Gq-Q209 L) and $3.8 \mathrm{nM}$ (Gq-R1830). Representative means and SEMs were calculated from triplicate values from one of at least three independent experiments for each mutant. $B$, effects of FR on UM cell lines. Growth of UM cell lines driven by constitutively active Gq or G11 mutants was inhibited by FR with IC50s between $0.5 \mathrm{nM}$ (MP41) and $38 \mathrm{nM}$ (Mel270). A UM cell line (OCM-1A) driven by constitutively active BRAF(V600E) was unaffected by FR. Representative means and SEMs were calculated from triplicate values from one of at least three independent experiments for each cell line. $C$, effect of FR on apoptosis and cell cycle progression of UM cell lines analyzed by flow cytometry. Induction of apoptosis was indicated by increased proportion of cells with sub-G1/G0 DNA content. Cell cycle arrest was indicated by decreased proportion of cells with S- and G2/M-phase DNA content. Representative means and SEMs calculated from triplicate values from one of at least three independent experiments for each cell line. 
before (17-19), demonstrating the selectivity of FR for oncogenic Gq/11 in UM.

\section{FR redifferentiates class 1 but not class 2 UM cells}

Class 1 and class 2 primary UM tumors have low $(\sim 5 \%)$ and high $(\sim 90 \%)$ probability of metastasis, respectively $(33,34)$. Class 1 UM tumors express the metastasis suppressor, BAP1, encoding an enzyme that regulates gene expression epigenetically by deubiquitinating histone $\mathrm{H} 2 \mathrm{~A}$. Class $2 \mathrm{UM}$ tumors are BAP1-deficient (35) and possess additional genetic defects rendering them prone to metastasis (32). Almost all UM cell lines are derived from class 1 tumors (36), raising the crucial question of whether both classes of primary UM tumor cells can be targeted by FR.
We addressed this question initially by analyzing MP41 (class 1; $\mathrm{BAP}^{+}$) and MP46 (class 2; BAP1-deficient) cell lines, which were established originally from patient-derived xenografts (PDX) of primary UM tumors (37). We found that FR arrested growth and cell cycle progression of both of these cell lines without triggering significant apoptosis (Fig. 1, B and C).

Next, we used transcriptional analysis to determine whether FR could redifferentiate MP41 (class 1) and MP46 (class 2) cells into melanocytic-like cells, as we had shown previously with two other class 1 UM cell lines (92.1 and Mel202) (17). MP41 and MP46 cells both responded transcriptionally to FR, as indicated by multidimensional scaling analysis of RNAseq data (Fig. 2A). However, a cluster of genes was downregulated preferentially in class $1 \mathrm{MP} 41$ cells (circled in Fig. 2B), as
A

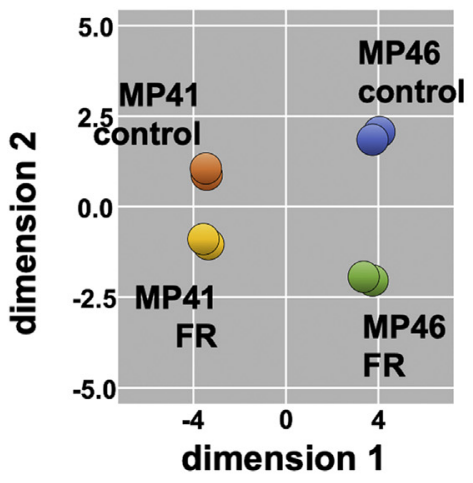

D

D

\section{G11(Q209L) / Class1}
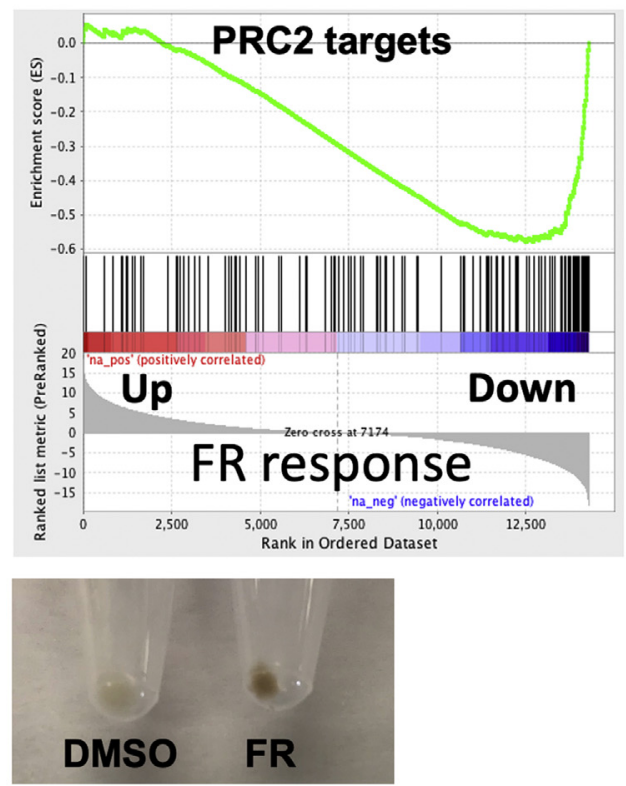

B

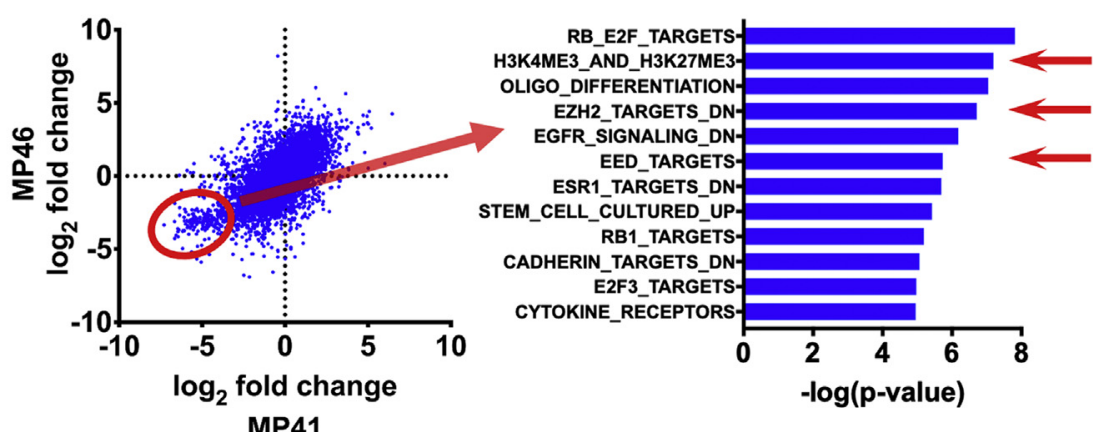

MP41

C
MP46

Gq(Q209L) / Class2
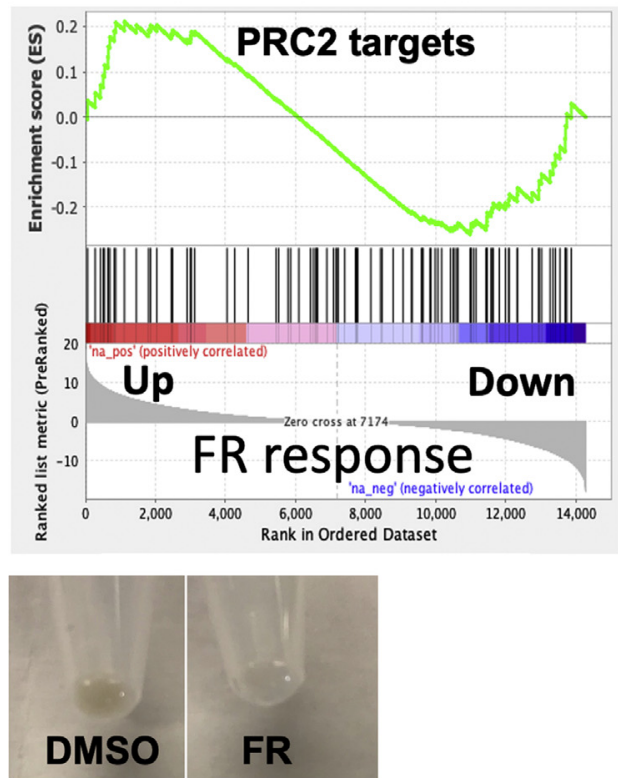

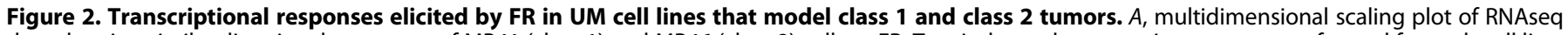

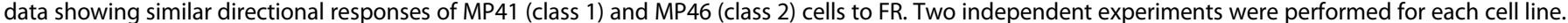

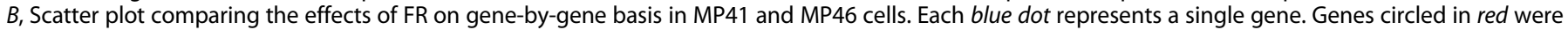

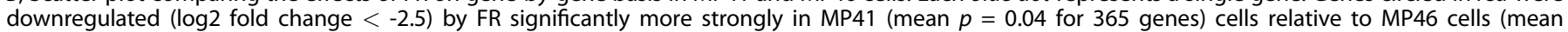

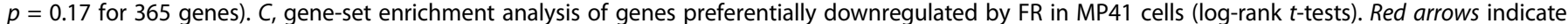

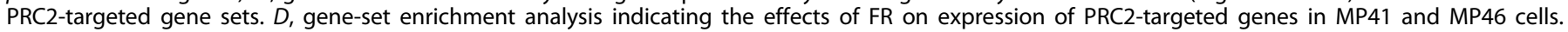

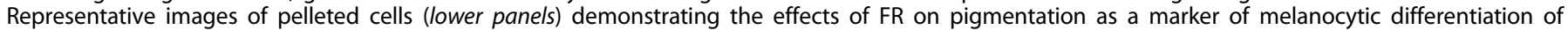
MP41 and MP46 cells. 
A

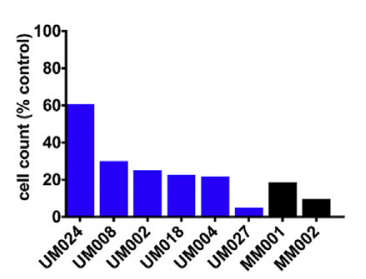

$\mathrm{C}$

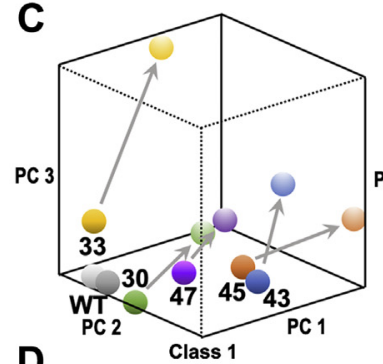

B

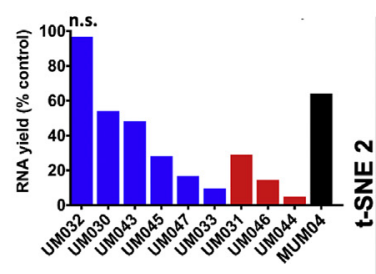

E

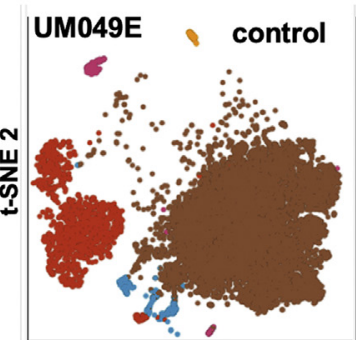

t-SNE 1
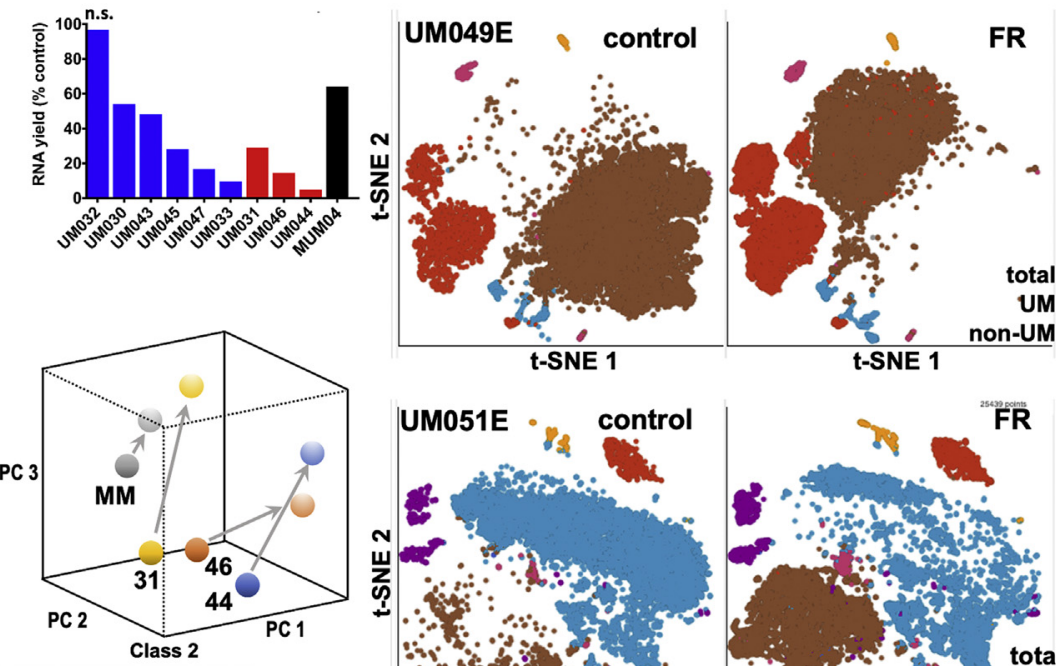

Cells
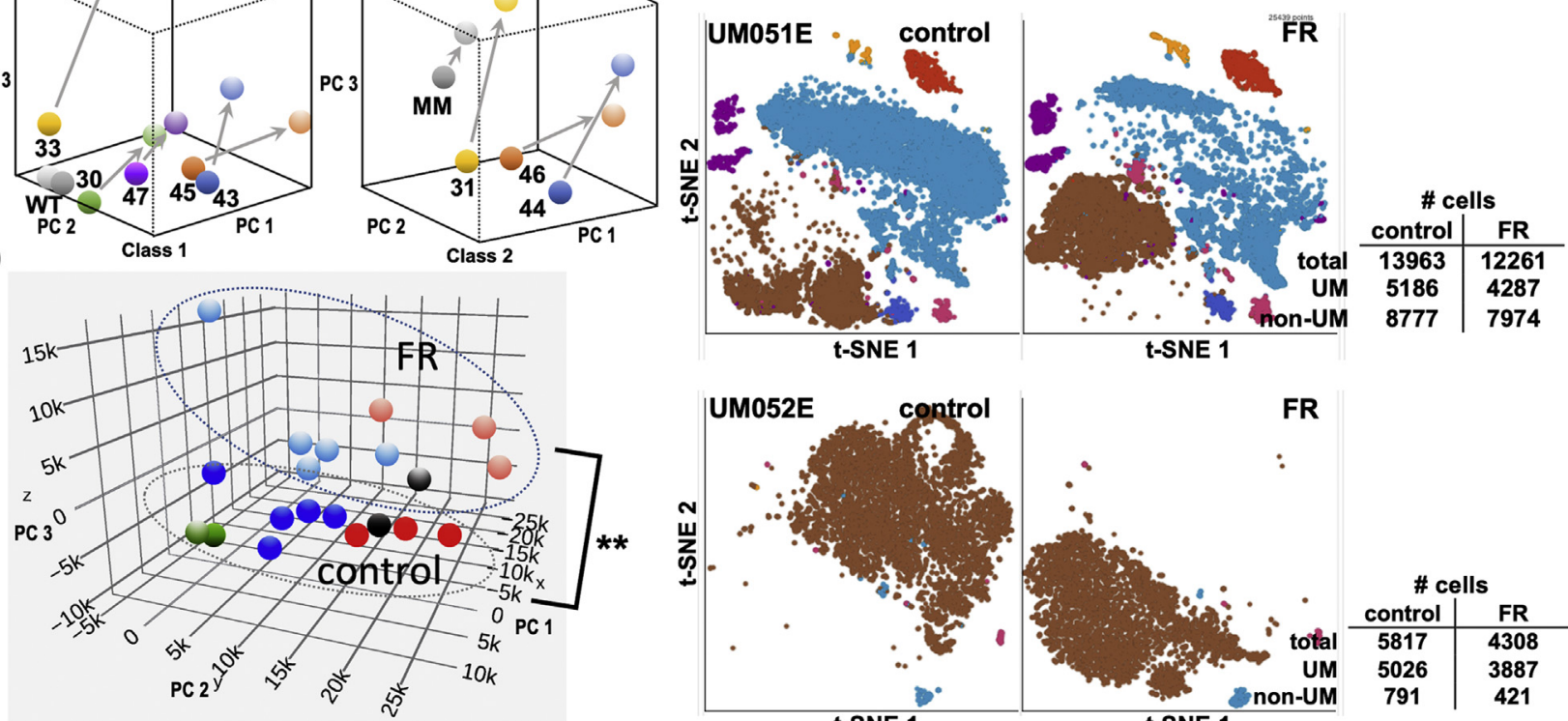

t-SNE 1

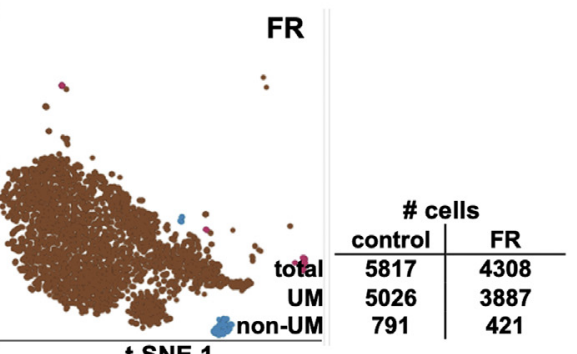

Figure 3. Response of human UM tumor biopsy samples to FR ex vivo. $A$, effects of FR on cell abundance in self-renewing cultures from UM patient biopsies. Human tumor fine-needle biopsy samples of the indicated UM tumors were established in culture and split equally. Cells were counted after 7 days of culture upon treatment with vehicle or FR (100 nM). Each bar indicates an individual tumor sample. $B$, effect of FR on total RNA yield from UM patient biopsies. The indicated human UM biopsy samples from class 1 and class 2 primary UM tumors and a liver metastasis were dissociated, split equally, and treated immediately for 7 days with vehicle or FR $(100 \mathrm{nM})$. The effect of FR relative to vehicle controls on the yield of total RNA as a marker of cell number is shown. Each bar indicates an individual tumor sample. One biopsy sample (UM032) expressed wildtype Gq and G11 and was unaffected by FR. In panels $A$ and $B$, class 1 tumor samples are blue; class 2 tumor samples are red; metastatic tumor sample is gray. C, effect of FR on global gene expression in freshly isolated UM biopsies detected by RNAseq. Principal component analysis (PCA) of RNAseq data from class 1 (including the Gq/11-wild-type sample: WT) and class 2 (including metastatic sample: MM) human tumor biopsy samples. Arrows indicate the directional effect of FR on gene expression for each biopsy sample. $D$, combined PCA analysis of the effects of FR on gene expression in UM biopsy samples shown in panel C. Class 1 tumor samples are blue; class 2 tumor samples are red; metastatic tumor sample is gray; unresponsive wild-type Gq/G11 sample (UM032) is green. Correlations of principal component 1 (x-axis) and principal component 3 (z-axis) with FR-treatment were significant (paired $t$-tests with Holm-Sídák correction, $p<0.01:{ }^{*}$ ). E, FR elicits transcriptional responses in UM tumor but not stromal cells as detected by single-cell RNAseq. Two-dimensional t-SNE plots of single-cell scRNAseq data are shown for each of the three indicated primary UM tumor samples obtained after enucleation, and treated 7 days with vehicle or FR $(100 \mathrm{nM})$. Individual cell types are color coded in each panel. t-SNE plots of data obtained after vehicle (left panel) and FR (right panel) treatment of the indicated tumor samples are shown. The transcriptional profile of melanoma cells from a given tumor overlap little in vehicle- and FR-treated samples, indicating marked transcriptional response to FR. The transcriptional profile of each nonmelanoma cell type from a given tumor overlap extensively in vehicle- and FR-treated samples, indicating little transcriptional response to FR.

revealed by analyzing transcriptional responses on a gene-bygene basis. We defined this cluster as genes with $\log 2$ fold change in MP41 cells $<-2.5$ in response to FR, which identified 365 genes that were more significantly downregulated in MP41 cells (mean $p=0.04$ ) than MP46 cells (mean $p=0.17$ ). Many genes in this cluster are targets of epigenetic regulation by polycomb repressive complex 2 (PRC2), based on gene set analysis (H3K27trimethylation, EZH2; Fig. 2C). This PRC2targeted gene cluster was coordinately repressed by FR in class 1 MP41 cells as compared with class 2 MP46 cells (Fig. 2D). Consistent with these distinctions, FR induced pigmentation, a hallmark of melanocytic differentiation, of
MP41 but not MP46 cells (Fig. 2D). Indeed, our prior studies had shown that PRC2 activity is required for FR to reinstate melanocytic differentiation of two other class $1 \mathrm{UM}$ cell lines (92.1 and Mel202) (17). Therefore, the ability of FR to promote melanocytic redifferentiation of primary UM tumors may depend on tumor class.

\section{FR targets UM cells from primary and metastatic tumors}

Because UM tumor cell lines do not fully recapitulate important properties of UM tumors, we determined whether FR can target UM cells obtained from patient biopsies of primary and metastatic tumors. We began by preparing 
separate self-renewing cultures of UM tumor cells isolated from fine-needle biopsies of class 1 primary tumors from six patients and core biopsies of liver metastases from two patients. We found that all eight individually cultured-cell samples responded to $\mathrm{FR}$, as indicated by lower cell numbers relative to vehicle controls (Fig. $3 A$ ), and we noted, as shown before $(36,37)$, that class 2 primary UM tumor samples proliferated too poorly in vitro to establish self-renewing cultures. Accordingly, each time thereafter we obtained biopsy samples from additional patients, we prepared short-term cultures and analyzed the effects of FR. A total of ten biopsy samples (Table S1), including class 1 and class 2 primary tumors and a liver metastasis, were analyzed. Cultured cells from nine of these patient samples responded to FR relative to vehicle controls, as indicated by decreased yields of total RNA as a marker of cell number (Fig. $3 B$ ). The single nonresponding sample (UM032; Fig. 3B) expressed wild-type Gq and G11 (Table S1) and thus was driven by other oncogenes whose identities were not determined. For the nine FR-responsive UM tumor samples, which included class 1 and class 2 primary tumors and a liver metastasis, we found that FR caused similar directional effects on gene expression, as revealed by unsupervised principal component analysis (PCA) (Fig. 3C). Interestingly, the principal component that distinguished class 1 from class 2 and metastatic tumor cells (Fig. 3D; y-axis) (33) was relatively unaffected by FR (Fig. $3 D$ ), suggesting that FR did not cause class 2 UM tumor samples to lose their class identity and become more class 1-like. The one UM tumor sample that expressed wild-type Gq and G11 did not show significant directional effects of FR on gene expression (Fig. 3C). Together, these results demonstrated that, regardless of tumor class, primary and metastatic UM tumor samples driven by mutant $\mathrm{Gq} / 11$ responded ex vivo to $\mathrm{FR}$.

Because patient-derived UM tumor biopsies include cancer and stromal cells, we determined which cell types were targeted by FR, as indicated by their relative abundance and transcriptional responses revealed by single-cell RNAseq (scRNAseq). Primary tumor samples from three UM patients undergoing enucleation were used to obtain sufficient quantity of cells for these experiments. Dissociated UM tumor and stromal cells were treated 7 days with FR at a concentration $(100 \mathrm{nM})$ that strongly inhibited all oncogenic Gq/ 11 mutants (Fig. 1A). Transcriptional signatures readily distinguished UM cells from stromal cell types (Fig. $3 E$ ). In all three biopsy samples, FR modestly reduced the abundance of UM cells and had little effect on the abundance of non-UM cells (Fig. 3E). FR therefore did not cause extensive death of UM cells.

Further analysis of scRNAseq data indicated that UM cells, but not stromal cells, displayed striking transcriptional responses to FR. As visualized by t-SNE plots of scRNAseq data (Fig. 3E), FR dramatically shifted the transcriptional profile of UM cells in all three biopsy samples. These changes in UM gene expression in tumor samples from enucleated patients were analogous to those observed previously by bulk RNAseq of small biopsy samples (Fig. 3D). In contrast, FR did not significantly affect the transcriptional profiles of stromal cells in all three biopsy samples (Fig. 3E). UM tumor cells therefore were targeted transcriptionally by FR.

Transcriptional analysis also indicated that FR inhibited cell cycle progression of patient-derived UM tumor cells. FRevoked changes in gene expression detected by scRNAseq in UM cells from enucleated tumors and bulk RNAseq of small biopsies were similar, as shown by hallmark enrichment analysis (Fig. 4A). In both cases, targets of E2F transcription factors (arrows in Fig. 4, $A$ and $B$ ) were among the gene sets prominently downregulated by FR. Moreover, FR treatment of class 1 and class 2 biopsy samples downregulated expression of the E2F transcription factors E2F1, E2F2, and E2F8 (Fig. 4C) and upregulated expression of the cell cycle inhibitors RB1 and p130 (38). In sum, inhibition of oncogenic Gq/11 by FR upregulated $\mathrm{Rb}$ expression and downregulated E2F expression, thereby contributing to inhibition of UM cell cycle progression (Fig. 4D).

Next, we investigated whether tumor class, which predicts metastatic potential and patient prognosis, determines whether FR evokes melanocytic redifferentiation of UM tumor cells in patient biopsies, as we had observed above with UM cell lines (Fig. 2). When we examined transcriptional responses of patient-derived class $1 \mathrm{UM}$ tumor cells to FR, we found strong enrichment of gene sets targeted by PRC2 (Fig. 4E). Nearly all members of the FR-responsive PRC2-targeted gene cluster that were identified in UM cell lines (Fig. 2) were also downregulated in class $1 \mathrm{UM}$ tumor biopsies (Fig. 4F). In contrast, many of these genes were upregulated, rather than downregulated by FR in class 2 UM tumor biopsies (Fig. 4F). Furthermore, melanocyte differentiation genes, including MITF, TYR, and TYRP1, were upregulated significantly by FR in class 1 relative to class 2 tumor biopsy samples (Fig. 4G). Therefore, FR treatment potentially could inhibit progression of class 1 primary UM tumors by promoting melanocytic redifferentiation.

\section{FR up- and downregulates YAP1-driven genes}

Prior studies of UM have shown that oncogenic Gq/11 signaling leads to activation of the YAP1 transcription factor $(19,20)$. We therefore determined whether YAP-targeted genes are coordinately downregulated by FR in biopsy samples of class 1 and class 2 primary UM tumors. In contrast to this expectation, we found that FR upregulated and downregulated YAP-targeted genes in nearly equal proportion (Fig. S2). The mechanisms responsible for the complex effects of FR on YAP-targeted genes are unknown, but potentially involve the combinatorial actions of other transcription factors with YAP1 on the promoters of these genes.

\section{A therapeutic window for targeting UM tumors with $F R$}

Because inhibition of $\mathrm{Gq} / 11$ activity by more than $50 \%$ is likely to be lethal, as indicated by gene dosage studies in knockout mice (29), we sought to define protocols and conditions under which chronic, systemic administration of FR is physiologically tolerated, so we could assess the therapeutic potential of FR in mouse xenograft models of UM. Based on 
A

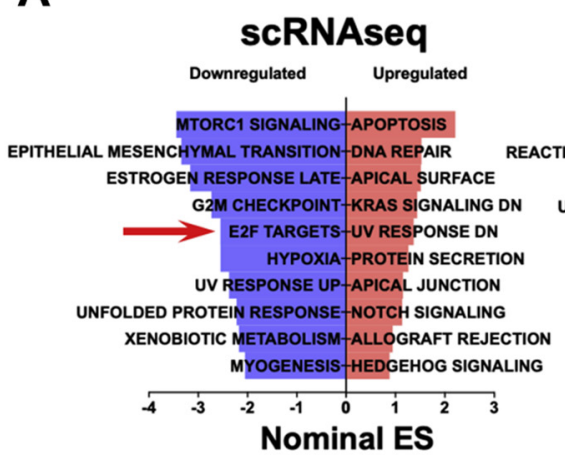

C

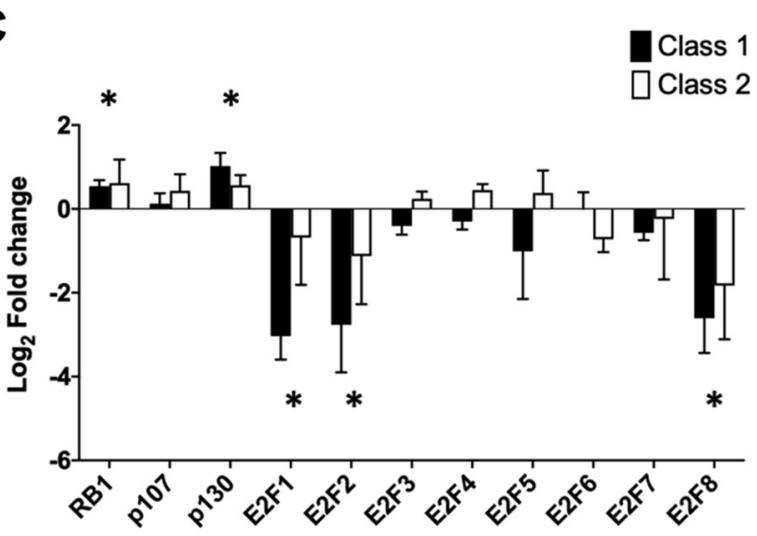

bulk RNAseq

Downregulated Upregulated

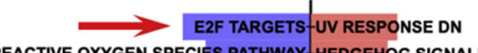
SPECIES PATHWAY HEDGEHOG SIGNALING G2M CHECKPOINT-TGF BETA SIGNALING UNFOLDED PROTEIN RESPONSE EMT

UV RESPONSE UP-WNT BETA CATENIN SIGNALING DNA REPAIR-NOTCH SIGNALING

PEROXISOME-P53 PATHWAY

ESTROGEN RESPONSE LATE ESTROGEN RESPONSE EARLY

XENOBIOTIC METABOLISM INFLAMMATORY RESPONSE PROTEIN SECRETION-ALLOGRAFT REJECTION

$\begin{array}{lllllll}-3 & -2 & -1 & 0 & 1 & 2 & 3\end{array}$

Nominal ES
B
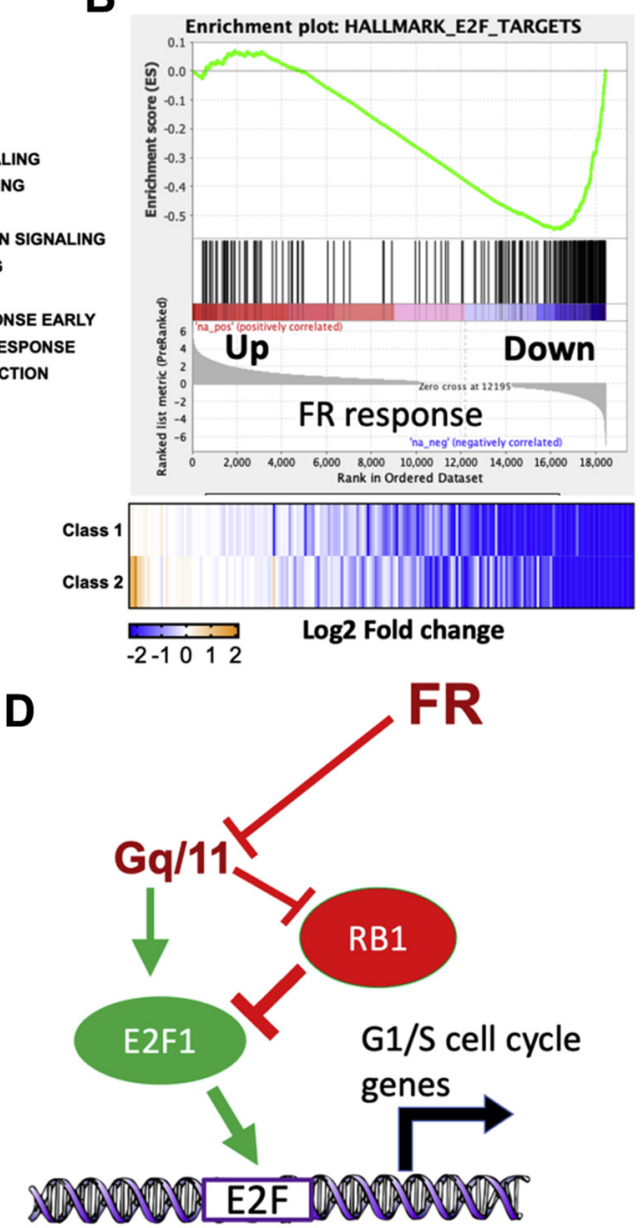

G
E

\section{Class 1 specific}

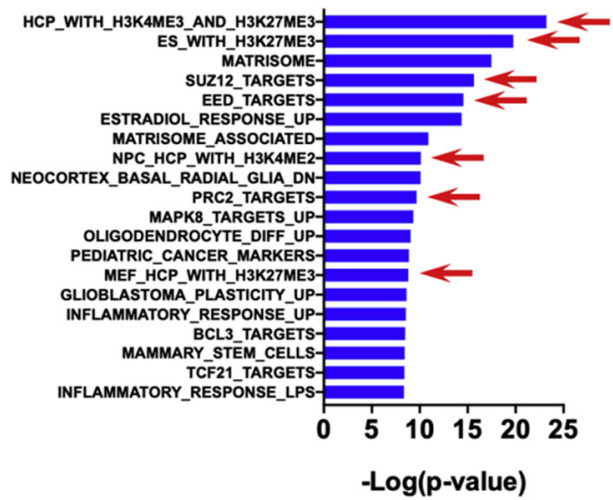

-Log(p-value)
$\mathbf{F}$

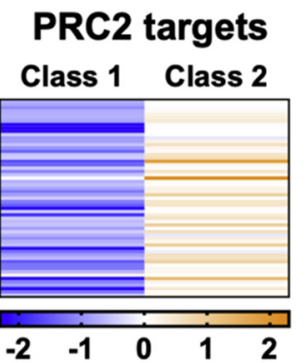

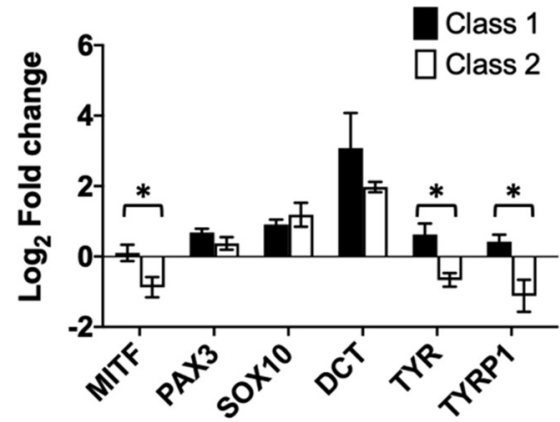

Figure 4. Pathway analysis of FR-induced transcriptional responses in UM. $A$, hallmark gene-set enrichment analysis of differential gene expression in response to FR. Left panel shows gene sets affected by FR in enucleation-derived UM cells identified by scRNAseq. Right panel shows gene sets affected by FR in nine biopsy samples described in Figure 3 as detected by RNAseq. Red arrow indicates that E2F target genes were significantly downregulated in both scRNAseq and bulk RNAseq datasets. $B$, enrichment plot of the E2F target hallmark gene set detected by bulk RNAseq. Heatmap below shows insignificant difference in response of this gene set to FR in class 1 versus class 2 biopsy samples. $C$, effects of FR on expression of Rb/E2F family members in class 1 and class 2 biopsy samples detected by RNAseq ( $107:$ RBL1; p130:RBL2). Asterisks indicate significant effect $(p<0.01$; paired $t$-test) relative to vehicle controls. Differences between class 1 and class 2 samples were insignificant. $D$, proposed model for cell cycle regulation by constitutively active Gq/11 and FR. Signaling by constitutively active $\mathrm{Gq} / 11$ leads to repression of Rb expression and induction of E2F expression that drives UM cell proliferation. FR attenuates these effects to arrest cell cycle progression. $E$, differential responses of class 1 and class 2 tumor biopsy samples to FR. Gene-set enrichment analysis identifies genes downregulated preferentially in class 1 relative to class 2 tumor biopsy samples (log-rank $t$-test). Red arrows indicate PRC2-specific gene sets. F, heat map showing effects of FR on PRC2-targeted genes in class 1 versus class 2 tumor samples. $G$, responses of melanocytic differentiation genes to FR in class 1 and class 2 tumor biopsy samples. Asterisks indicate statistically significant $(p<0.01$; paired $t$-test) effects of FR on the indicated melanocytic differentiation genes in class 1 and class 2 biopsy samples. 
prior studies examining the acute effects of FR or the closely related inhibitor YM-254890 (YM) (22, 24, 39), we administered FR by subcutaneous injection at $0.1-3.0 \mathrm{mg} / \mathrm{kg}$ on alternate days for 30 days to define an $\mathrm{LD}_{50}$ of $\sim 0.6 \mathrm{mg} / \mathrm{kg}$ in NOD-scid-gamma (NSG) mice used as xenograft recipients (Fig. 5A). We then determined the effect of FR administered chronically below its $\mathrm{LD}_{50}(0.1$ or $0.3 \mathrm{mg} / \mathrm{kg}$ by subcutaneous injection on alternate days) on several physiological parameters of NSG mice. Blood pressure, a sensitive indicator of Gq/ 11 activity (40), decreased significantly in conscious mice upon each injection of FR relative to vehicle controls (Fig. $5 B$ ), but rebounded to baseline within $24 \mathrm{~h}$ (Fig. 5C). Heart rate was affected insignificantly by FR (Fig. $5 B$ ). No evidence of anemia, monocyte or platelet deficiency (Table S2), or liver disfunction (Fig. 5D) was observed after treating NSG mice 30 days with FR at either dose relative to vehicle controls. Lastly, when administered for 1 week at the higher dose, FR had insignificant effect on the activity, exploratory behavior, or sensorimotor function of NSG mice (Table 1 and Fig. S3), even though $\mathrm{Gq} / 11$ are important regulators of nervous system
A
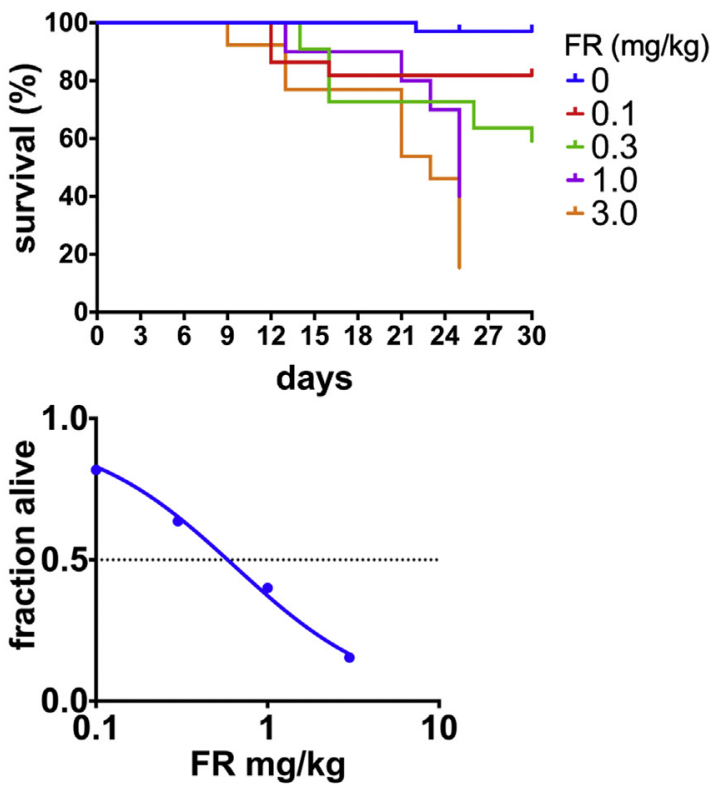

D

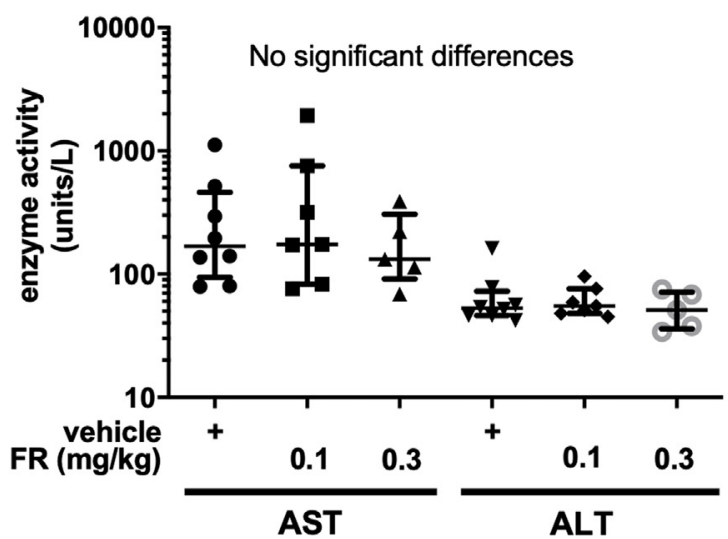

B
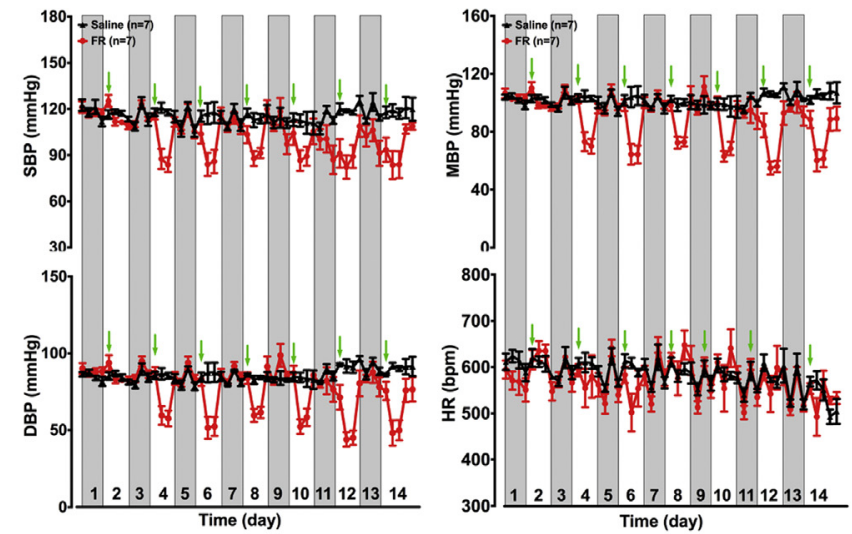

C
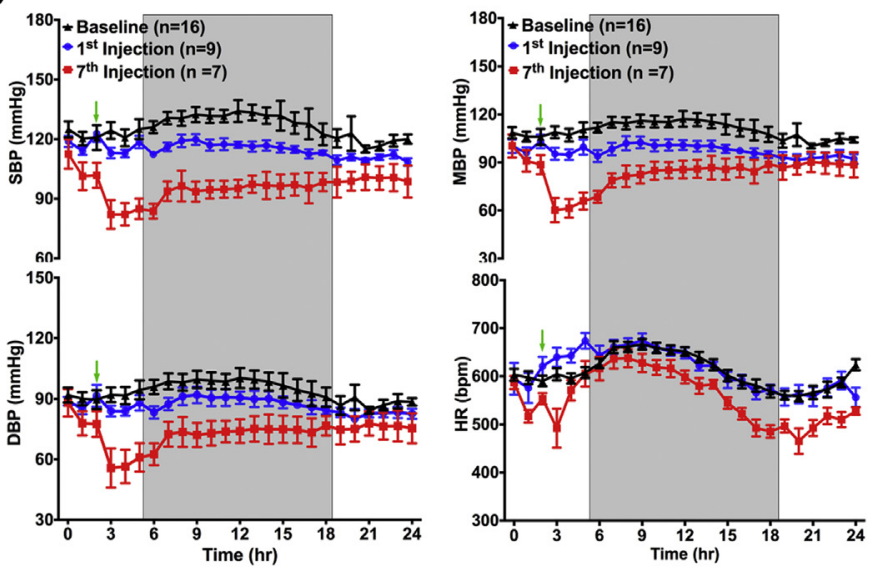

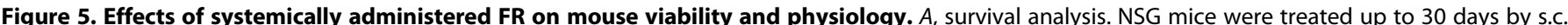

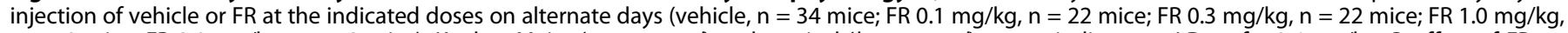

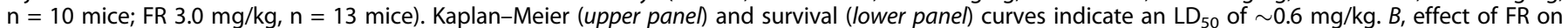

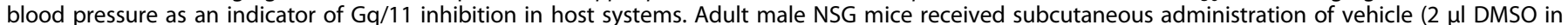

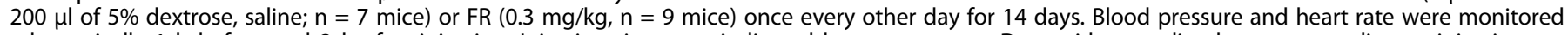

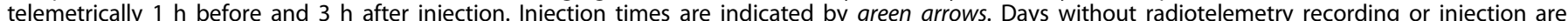

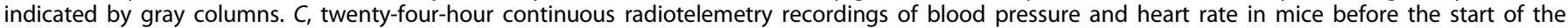

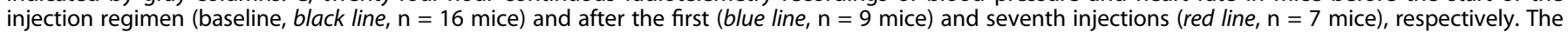

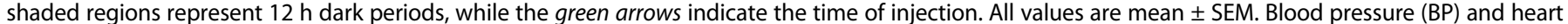

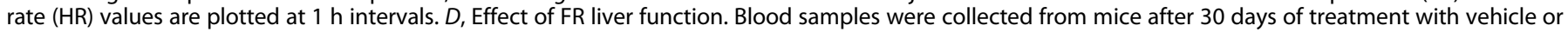

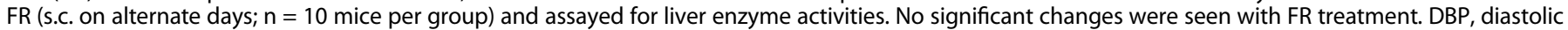
blood pressure; HR, heart rate; MBP, mean blood pressure; SBP, systolic blood pressure. 
Table 1

Effect of FR on mouse behavior

\begin{tabular}{|c|c|c|c|c|}
\hline Variable & CTL mean & TX mean & Diff \pm SEM & $p$ value \\
\hline Walking initiation (s) & 2.54 & 1.98 & $0.96 \pm 1.57$ & $p=0.3537$ \\
\hline Ledge (/60s) & 48.92 & 43.35 & $29.12 \pm 19.79$ & $p=0.4315$ \\
\hline Platform (/60s) & 22.97 & 25.74 & $4.4 \pm 18.55$ & $p=0.7294$ \\
\hline Pole (/120s) & 25.92 & 22.16 & $0.84 \pm 25.07$ & $p=0.5844$ \\
\hline Turn (/60s) & 23.73 & 15.97 & $-1.79 \pm 25.52$ & $p=0.5844$ \\
\hline 60 degree screen $(/ 60 s)$ & 11.43 & 10.68 & $7.88 \pm 3.54$ & $p=0.634$ \\
\hline 90 degree screen $(/ 60 s)$ & 16.98 & 16.49 & $7.04 \pm 9.93$ & $p=0.9049$ \\
\hline Inverted screen $(/ 60 \mathrm{~s})$ & 49.92 & 41.10 & $32.17 \pm 17.74$ & $p=0.1437$ \\
\hline Fine movements (/1h) & 1758 & 1549.00 & $209.1 \pm 115.5$ & $p=0.0861$ \\
\hline Total ambulations (/1h) & 756.5 & 674.3 & $-82.20 \pm 64.16$ & $p=0.2155$ \\
\hline Time rearing $(/ \mathrm{hr})$ & 688.5 & 583.3 & $105.1 \pm 81.22$ & $p=0.2111$ \\
\hline Center distance $(\mathrm{mm} / \mathrm{h})$ & 4288 & 3941 & $347.4 \pm 506.5$ & $p=0.5010$ \\
\hline Center time $(/ 1 \mathrm{~h})$ & 478.3 & 557.6 & $-79.30 \pm 112.8$ & $p=0.4905$ \\
\hline Center entries (/1h) & 263.9 & 236.1 & $27.81 \pm 27.70$ & $p=0.3281$ \\
\hline Total rest time $(\mathrm{s} / 1 \mathrm{~h})$ & 1504 & 1635 & $-130.6 \pm 118.7$ & $p=0.2847$ \\
\hline Total ambulations over time & 4539 & 4046 & $493.2 \pm 385.0$ & $p=0.2733$ \\
\hline Rearing frequency over time & 116.8 & 123.5 & $6.671 \pm 14.51$ & $p=0.8508$ \\
\hline
\end{tabular}

development and activity (40). Chronic systemic administration of FR below its $\mathrm{LD}_{50}$ therefore had modest or undetectable effects on physiological systems that potentially could limit dosing regimens in UM clinical trials.

Next, we determined whether FR delivered systemically at physiologically tolerated doses could inhibit growth of xenografted UM tumors driven specifically by oncogenic Gq/11. These experiments used MP41 and MP46 cells, for several reasons. These UM cell lines were isolated originally from patient-derived xenografts, provide models of class 1 and class 2 tumors, and, of the UM cell lines tested, responded to FR (cell cycle arrest, with little or no apoptosis) most like UM tumor cells from patient biopsies. We also used a UM cell line (OCM-1A) driven by BRAF(V600E) to determine whether effects of FR were specific for Gq/11-driven tumors. Accordingly, subcutaneous UM tumor xenografts of MP41, MP46, and OCM-1A cells were established, and vehicle or FR was administered systemically at low $(0.1 \mathrm{mg} / \mathrm{kg})$ or high $(0.3 \mathrm{mg} / \mathrm{kg})$ dose on alternate days by subcutaneous injection on the contralateral side. Tumor size was measured over time and tumor weight was determined at the end of the experiment. Relative to vehicle controls, overall survival of tumorbearing mice treated $\sim 30$ days with FR at low or high dose was respectively $85 \%$ and $71 \%$. Under these conditions, FR inhibited growth of MP41 tumors only at the high dose (Fig. 6A), but inhibited growth of MP46 tumors at either dose (Fig. 6A). FR had no effect on growth of BRAF(V600E)-driven OCM-1A tumors (Fig. 6A), demonstrating that FR did not affect processes such as tumor vascularization or perfusion that generically support tumor growth. Thus, FR specifically targeted both class 1 and 2 UM tumor xenografts, but only those driven specifically by oncogenic Gq/11.

To determine the extent to which FR delivered systemically at a therapeutically effective dose inhibited oncogenic $G$ protein signaling in vivo, we compared the effects of FR on Erk activity, one of several downstream pathways activated by oncogenic Gq/11 in UM (41), in vitro vs. in vivo (Figs. $6 B$ and S4). We used MP41 cells and tumors because, in the absence of FR, phosphorylated Erk was detected readily and reproducibly in vitro and in vivo, as compared with MP46 cells and tumors. As a reference for in vivo studies, we first determined the maximal extent that Erk phosphorylation in MP41 cells was inhibited by FR in vitro. At concentration of FR $(1 \mu \mathrm{M})$ that maximally suppressed reporter activity driven by oncogenic Gq or G11 (Fig. 1A), Erk phosphorylation in MP41 cells was reduced in vitro by $85 \%$ (Figs. $6 B$ and S4). In contrast, FR administered in vivo at a therapeutically effective dose ( $0.3 \mathrm{mg} / \mathrm{kg}$ on alternate days) for 26 days reduced Erk phosphorylation in G11-driven MP41 tumors in vivo by only 30\% (Figs. $6 B$ and S4). Thus, modulation rather than complete suppression of oncogenic G protein signaling in MP41 tumor xenografts was sufficient for FR to have therapeutic effect.

Lastly, we investigated whether FR caused durable arrest or regression of class $1 \mathrm{UM}$ tumor xenografts. In this experiment, mice were implanted with MP41 cells and allowed to form tumors. MP46 tumors were not studied because their slow growth made it difficult to determine whether FR potentially caused durable arrest or regression. We arrested MP41 tumor growth by treating animals with FR at an effective dose $(0.3 \mathrm{mg} / \mathrm{kg}$ s.c. on alternate days $)$ for 25 days (Fig. 6 C). FR treatment then was stopped. Tumors resumed growth 16 days later (Fig. 6C). Therefore, FR appeared to cause reversible rather than durable arrest or regression of MP41 tumors. Taken together, these results indicated the existence of a therapeutic window in which FR effectively targeted class 1 or class 2 UM tumor xenografts, while preserving sufficient function of $\mathrm{Gq} / 11$ in host tissues required to support essential physiological processes and viability.

\section{Discussion}

\section{Targeting UM tumors with FR}

Here we have provided several new lines of evidence that support the therapeutic potential of $\mathrm{Gq} / 11$ inhibitors such as FR for UM. Our findings indicate that the vast majority of primary and metastatic UM tumors potentially can be targeted by FR. We found that all constitutively active mutant forms of $\mathrm{Gq} / 11$ found in UM, which collectively drive tumorigenesis in $>90 \%$ of patients, are sensitive to FR. Moreover, FR can target 
A
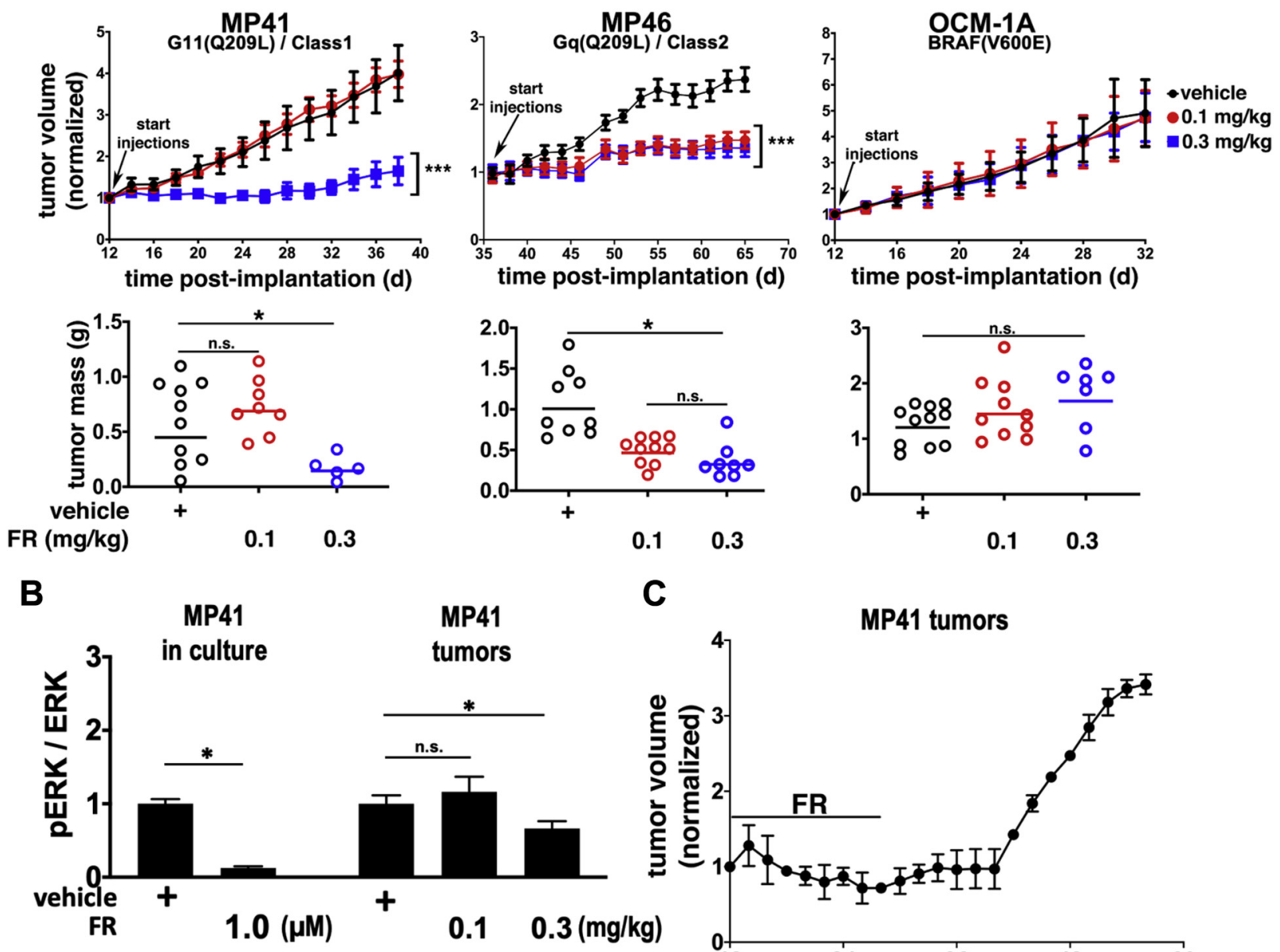

C

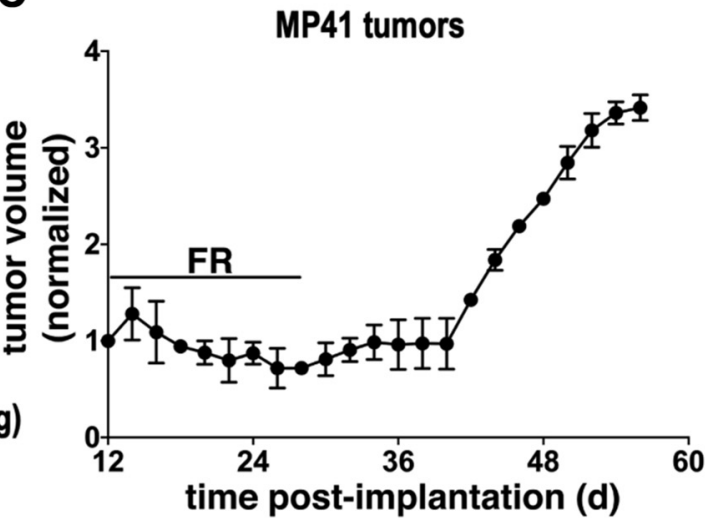

Figure 6. FR inhibits growth of xenografted class 1 and class 2 UM tumors. $A$, tumor volume (upper panels) as a function of time and dose of FR administered by s.c. injection of mice ( $n=9-11 /$ group) on alternate days. Effect of FR on tumor weight (lower panels) measured in surviving mice at the end of the experiment. $B$, effect of FR on Erk phosphorylation as a marker of oncogenic G11 signaling in MP41 cells in vitro versus tumors from animals treated with vehicle or FR at the indicated doses. Shown are the effects of FR on pERK: total Erk ratios relative to vehicle controls ( $n=3$ independent cell cultures/ condition; $\mathrm{n}=3$ independent tumors/condition); data shown are representative of three independent determinations, each performed with three cell culture and tumor replicates per condition. $C$, durability of MP41 tumor response to FR. MP41 tumor-bearing mice $(n=4)$ were treated systemically with FR for 28 days, after which time FR treatment was stopped. Tumors resumed growth 16 days after FR treatment was withdrawn. Results shown are representative of two independent experiments. Asterisks indicate $p<0.05$ (t-test) in panels $A$ and $B$.

Gq/11-driven UM tumor cells from patient biopsies of primary ocular tumors with low or high metastatic potential and liver metastases. And lastly, although FR does not discriminate between wild-type and oncogenic Gq/11, and host Gq/11 activity is physiologically essential, we were able to identify a therapeutic window in which FR strongly inhibited tumor growth without causing large negative effects on viability or physiology. UM tumors therefore appear to be particularly vulnerable to FR.

The vulnerability of UM tumors to FR may occur because the signaling networks downstream of oncogenic Gq/11 must be maintained above a high threshold. In support of this hypothesis, we found that a therapeutically effective dose of FR strongly inhibits UM tumor growth but only modestly reduces oncogenic G11-driven Erk phosphorylation in UM tumors. Because nearly complete inhibition of Erk phosphorylation by
FR was not necessary to inhibit UM tumor growth, Erk likely cooperates with other signaling pathways downstream of oncogenic Gq/11 (42) to sustain UM tumors. Indeed, Gq/11 and MEK inhibitors recently have been shown to synergize and regress UM tumor xenografts (30), suggesting that synergism might improve efficacy or safety of Gq/11 inhibitors such as FR as therapeutic agents for UM.

\section{Therapeutic implications}

Our results suggest several ways that $\mathrm{Gq} / 11$ inhibitors such as FR could be explored therapeutically to treat UM patients, either alone or in conjunction with current standards of care. Primary UM tumors usually are treated by external beam or plaque irradiation of the eye (12) to arrest growth, induce tumor cell death, and preserve vision, although these interventions on their own do not improve prognosis for 
metastasis (13). Once metastatic UM occurs in the liver, therapy involves mechanical isolation of the hepatic vasculature by embolization and percutaneous infusion of high-dose chemotherapeutics (43) or radiotherapy (44) above what would be tolerated systemically (45). Thus, targeting delivery of FR to primary UM tumors by intravitreal injection, or hepatic metastases by chemoembolization, could be considered. Targeted delivery might be required to reduce the impact of FR on viability, blood pressure, or other dose-limiting physiological processes, or allow FR to be delivered at higher doses in an effort to promote durable arrest or tumor regression. If FR proves to have limited efficacy in monotherapy, it could be combined with standard-of-care interventions to treat primary and metastatic UM. Intravitreal injection of FR also might interfere with progression of very early-stage lesions to class 2 tumors that have high metastatic potential by promoting melanocytic differentiation, as we have shown for class 1 UM tumor cells ex vivo. Similarly, because intraocular nevi are relatively common, express constitutively active $\mathrm{Gq} / 11$, and can progress to early stage melanomas (46), intravitreal injection of FR might interfere with this process and reduce the incidence of UM.

UM is only one of several diseases that potentially could benefit from targeted treatment with FR. Constitutively active mutant forms of Gq/11 occur in $~ 5 \%$ of all cancers, including melanocytic neoplasms of the central nervous system (4), mucosal melanoma (5), choroidal hemangiomas $(8,11)$, and hepatic small vessel neoplasms (6), as well as in Sturge-Weber syndrome (7), certain forms of hypercalcemia and hypocalcemia (9), and autosomal dominant hypoparathyroidism (10). Treatment regimens for all of these conditions potentially could be improved by novel therapeutic approaches employing FR.

\section{Experimental procedures}

\section{Reagents}

FR900359 (FR) was purified from Ardisia crenata according to published methods (16). SRE.L assays used a firefly luciferase reporter driven by the Gq/11-dependent SRE promoter and were performed as described (47). Firefly luciferase activity was normalized to Renilla luciferase expressed from a constitutive promoter.

\section{Cell culture assays}

Cells were cultured at $37 \mathrm{C}^{\circ}$ in $5 \%$ CO2. Human 92.1 (RRID:CVCL_8607), Mel270 (RRID:CVCL_C302), and OCM1A (RRID:CVCL_6934) UM cells were derived by and the generous gifts of Drs. Martine Jager (Laboratory of Ophthalmology, Leiden University), Bruce Ksander (Schepens Eye Institute, Massachusetts Eye and Ear Infirmary), and June KanMitchell (Biological Sciences, University of Texas at El Paso), respectively. The human PDX cell lines MP41 (ATCC Cat\# CRL-3297, RRID:CVCL_4D12) and MP46 (ATCC Cat\# CRL3298, RRID:CVCL_4D13) cell lines were purchased from ATCC (Manassas, VA). Cell lines were grown in RPMI 1640 medium (Life Technologies, Carlsbad, CA) supplemented with
FBS and antibiotics. Cell viability was measured using a watersoluble tetrazolium salt, WTS-8 (Bimake, Houston, TX), following the manufacturer's protocol. Flow cytometry for analysis of cell proliferation and apoptosis was performed at the Siteman Cancer Center Flow Cytometry Core on a FACScan analyzer (BD Biosciences, San Diego CA, USA) using a standard propidium iodide staining protocol as described previously (48).

\section{Patients and biopsy collection}

Human UM biopsy or enucleation samples were obtained with patient written informed consent and with approval of the institutional review board of Washington University in St Louis. Fine-needle aspiration biopsies of primary UM tumors and ultrasound-guided core biopsies of UM liver metastases were performed as part of standard of care, which also collected biopsies for cytological evaluation of tumor cells and molecular classification (Castle Biosciences). For enucleation samples, after excision, eyes were transilluminated to localize the tumor mass and then opened opposite the tumor. Vitreous was carefully removed and tumor samples were collected through the retina from the tumor apex. All samples were collected directly into growth medium in the operating room before being transported to the laboratory. Primary uveal melanoma cells were divided equally into two wells of fibronectin-covered 6-well tissue culture plates and grown in $5 \%$ CO2 in MDMF medium, which consists of HAM's F12 (Lonza, Walkersville MD, USA) supplemented with $1 \mathrm{mg} / \mathrm{ml}$ BSA (Sigma-Aldrich, St Louis MO, USA), 2 mM L-glutamine (Lonza), 1X SITE (Sigma-Aldrich), 1x B27 (Gibco, Carlsbad CA, USA), 20 ng/ml bFGF (PeproTech Inc, Rocky Hill NJ, USA), and $50 \mu \mathrm{g} / \mathrm{ml}$ Gentamicin (Sigma-Aldrich) (49). Cells were allowed to attach to the substrate overnight before fresh medium was added to each well containing either 100 nM FR or an equal volume of vehicle (DMSO).

\section{Bulk RNAseq}

MP41 and MP46 cells were treated with 100 nM FR or vehicle (DMSO) in RPMI growth medium and collected after 3 days of treatment. FNAB samples were treated with $100 \mathrm{nM}$ FR or vehicle in MDMF medium and collected after 7 days of treatment. RNA was isolated using the RNeasy Mini Kit (Qiagen) following the manufacturer's protocol and including the optional DNase I treatment step. RNA quality was assessed on a Bioanalyzer 2100 (Agilent Technologies, Santa Clara, CA, USA). mRNA was extracted from total RNA using a Dynal mRNA Direct kit, fragmented, and reverse transcribed to double-stranded cDNA with random primers before addition of adapters for library preparation. Library preparation and HiSeq2500 sequencing were performed by the Washington University Genome Technology Access Center (gtac.wustl. edu). FastQ files were aligned to the transcriptome and the whole genome with STAR. Biologic replicates were simultaneously analyzed by edgeR and Sailfish analyses of gene-level/ exon-level features. Unexpressed genes and exons were removed from the analyses. Unsupervised principal component analysis was generated in Bioconductor using edgeR. 
Direct comparison of FR response in MP41 versus MP46 cells was used to identify MP41-specific genes, and this list was used as signature gene sets for Gene Set Enrichment Analysis (GSEA) (50).

\section{Single-cell RNAseq}

Single-cell RNAseq (scRNAseq) was performed using the 10X Genomics platform. Single-cell suspensions were counted using a hemocytometer and adjusted to $1000 \mathrm{cells} / \mu \mathrm{l}$. Sample processing through 10X Genomics and NextSeq 500 (Illumina) sequencing was performed by the Washington University Genome Technology Access Center (gtac.wustl.edu). Raw base call files were analyzed using Cell Ranger v.3.0.2. The filtered counts data from all six samples were combined in Partek Flow (www.partek.com). Samples were filtered on total reads, detected features, and mitochondrial content per cell to remove cell doublets and apoptotic debris. Unexpressed features were removed, and samples were normalized and log transformed.

PCA was used to reduce dimensionality, and the first 20 principal components were further analyzed by graph-based (Louvain) clustering and t-SNE. The resulting 24 clusters were visualized in a two-dimensional t-SNE representation and were annotated to known biological cell types using canonical marker genes. The following cell types were annotated (selected markers are listed): Lymphocytes (CD3D, CD3G, IL7R); macrophages (C1QA, C1QB, C1QC); dendritic cells (CD1C and lack of expression of C1QA, C1QB and C1QC); retinal cells (SYNGR1, NPTX1); photoreceptors (GNAT1, GNGT1); RPE(PAX6, KRT8, KRT18); melanoma (DCT, TYRP1, PMEL).

\section{Mice}

All experiments were performed using the NOD scid gamma (NOD.Cg-Prkdc ${ }^{\text {scid }}$ Il2rg ${ }^{\text {tm1 } 1 \text { Wil }} /$ SzJ: NSG) mice purchased from The Jackson Laboratory (\#005557). All animal experiments were performed under protocols approved by the Animal Studies Committees of Washington University in St Louis School of Medicine and Drexel University College of Medicine. FR was prepared from a $15 \mathrm{mM}$ stock solution in DMSO at doses of $0.1,0.3,1.0$, or $3.0 \mathrm{mg} / \mathrm{kg}$ and dextrose at a final volume of $200 \mu \mathrm{l}$. FR was administered subcutaneously every other day. Animals were randomly grouped $(n=10$ / group) for placebo (1\% DMSO) or FR treatments. MP41, MP46, and OCM1A cells were inoculated subcutaneously into the flanks of 5-week-old male NSG mice. Cells were prepared at a concentration of $2 \times 10^{6}$ cells $/ 100 \mu \mathrm{l}$ in a solution of icecold PBS/Matrigel Matrix (50/50 v/v) (Fisher Scientific \# CB40234 A). Primary tumors were allowed to grow to $\sim 30 \mathrm{~mm}^{2}$ basal area based on measurements taken with calipers and using the formula $(\mathrm{L} \times 2 \mathrm{~W}) / 2$ where $\mathrm{L}$ and $\mathrm{W}$ are the longest and the shortest basal diameters of the tumor, respectively, before beginning treatment with FR. Tumors reaching a maximum length of $2 \mathrm{~cm}$ were defined as censored end points. At censored end points or at the end of each time course, mice were euthanized, and the tumors were removed using sterile surgical technique. Excised tumors were weighed to determine end-point mass and snap-frozen in liquid nitrogen for later use.

\section{Immunoblotting}

For tumor samples, approximately $100 \mathrm{mg}$ of tumor was homogenized on ice in 1X Cell Lysis Buffer (Cell Signaling Technology, catalog\# 9803) supplemented with $1 \mathrm{mM}$ PMSF. For cultured cells, cells were grown in $10 \mathrm{~cm}$ dishes and treated with DMSO or $1 \mu \mathrm{M}$ FR for $18 \mathrm{~h}$, and then lysed in $1 \mathrm{X}$ Cell Lysis Buffer. Lysates were sonicated on ice for $2 \mathrm{~min}(30 \mathrm{~s}$ on, $30 \mathrm{~s}$ off, $60 \% \mathrm{~A}$ ), rotated end-over-end for $30 \mathrm{~min}$, and cleared by centrifugation at $16,000 \times g$ for $15 \mathrm{~min}$. Protein concentration was determined using Bio-Rad Protein Assay Dye Reagent (Bio-Rad, catalog\# 5000006). In total, $15 \mu \mathrm{g}$ of tumor protein was resolved on 12\% SDS-PAGE gels and transferred to Immobilon-FL PVDF membrane (Millipore, catalog\# IPFL00010). Membranes were blocked with 5\% (w/v) BSA in TBST [20 mM Tris pH 7.6, $137 \mathrm{mM} \mathrm{NaCl,} \mathrm{0.1 \%} \mathrm{v/v}$ Tween 20] and incubated with primary antibodies [Phosphop44/42 MAPK (Erk1/2) Cell Signaling Technology, catalog\# 4370S lot\#24 and p44/42 MAPK (Erk1/2) Cell Signaling Technology catalog\# 9107S lot\#10]. Membranes were washed with TBST at least three times and incubated with IRDye 680-coupled goat anti-rabbit (LI-COR, catalog\# 926-68,071, lot\# C90618-09) and IRDye 800 goat anti-mouse (LI-COR, catalog\# 926-32,210, lot\# C91210-09 antibodies (LI-COR Biosciences). After incubation, membranes were washed at least three times with TBST, and signals were detected using Odyssey model 9120 imaging system (LI-COR Biosciences).

\section{Mouse radiotelemetry}

Blood pressure and heart rate responses to chronic administration of vehicle or FR900359 were monitored by radiotelemetry in conscious mice by following previously described procedures $(24,51)$. Briefly, adult NSG male mice were implanted with a radiotelemetry pressure-sensing catheter in the right carotid artery under isoflurane (3\% mixed with 95\% oxygen) anesthesia. The body of the transmitter (HD-X10, Data Science International (DSI), St Paul, MN) was tunneled into a subcutaneous pouch on the left flank of the animal. The mice were allowed to recover from the surgery for 1 week after which baseline blood pressure and heart rate were recorded for $24 \mathrm{~h}$, followed by alternating days of subcutaneous administration of vehicle or FR. After the 24-h baseline recordings, the mice were randomly assigned to two groups: one group receiving FR $(0.3 \mathrm{mg} / \mathrm{kg}$ in dextrose, s.c.) and the other group receiving equal amounts of vehicle $(2 \mu \mathrm{l}$ DMSO in $200 \mu \mathrm{l}$ of $5 \%$ dextrose, s.c.). Daily recordings were conducted from 1 to 5 PM for 14 days, and FR or vehicle was administered every other day at $3 \mathrm{PM}$. Continuous 24-h BP recordings were conducted during the first injection and seventh injection of FR. Data was acquired and analyzed using DSI Ponemah software version 6.5. Averages of continuous systolic blood pressure (SBP), diastolic blood pressure (DBP), mean blood 
pressure (MBP), and heart rate (HR) recordings were plotted in $1 \mathrm{~h}$ intervals over $24 \mathrm{~h}$.

\section{Mouse behavioral tests}

Mice were moved to the animal facility and allowed to acclimate to the new environment for 1 week prior to behavioral testing. All behavioral testing was conducted during the light cycle, by a female experimenter blinded to experimental group. All equipment was cleaned with $2 \%$ chlorhexidine diacetate or $70 \%$ ethanol between animals.

\section{Open-field activity/exploratory behavior}

General activity levels and exploratory behavior were quantified over a $60-\mathrm{min}$ period in an open field $(47.6 \mathrm{~cm} \mathrm{~L} \mathrm{x}$ $25.4 \mathrm{~cm} \mathrm{~W} \times 20.6 \mathrm{~cm} \mathrm{H}$ ) constructed of Plexiglas and surrounded by computerized photobeam instrumentation (Kinder Scientific, LLC, Poway, CA). General activity variables (total ambulations, rearings, time at rest) along with measures of emotionality, including time spent, distance traveled, and entries made into the central zone, were analyzed.

\section{Sensorimotor battery}

Walking initiation, ledge, platform, pole, and inclined and inverted screen tests were performed as previously described (52). Time in each task was manually recorded. The average of two trials was used for analyses. Test duration was $60 \mathrm{~s}$, except for the pole test, which was extended to $120 \mathrm{~s}$. For walking initiation, time for an animal to leave a $21 \times 21 \mathrm{~cm}^{2}$ square on a flat surface was recorded. For ledge and platform tests, the time the animal was able to balance on an acrylic ledge $(0.75 \mathrm{~cm}$ wide and $30 \mathrm{~cm}$ high), and on a wooden platform $(1.0 \mathrm{~cm}$ thick, $3.0 \mathrm{~cm}$ in diameter and elevated $47 \mathrm{~cm})$, was recorded, respectively. The pole test was used to evaluate fine motor coordination by quantifying time to turn $180^{\circ}$ and climb down a vertical pole. The screen tests assessed a combination of coordination and strength by quantifying time to climb up or hang onto a mesh wire grid measuring 16 squares per $10 \mathrm{~cm}$, elevated $47 \mathrm{~cm}$ and inclined $\left(60^{\circ}\right.$ or $\left.90^{\circ}\right)$ or inverted.

\section{Statistical analyses}

All statistical analyses were performed using IBM SPSS software (v.24) and Graphpad Prism 8. For cell line data, all experiments were performed in triplicate at least three times on different days. Means and standard errors were computed from all cell line data and $t$-tests were used to determine significance. For significance analysis of PCA data, correlation of each principal component with FR treatment was calculated using paired $t$-tests with Holm-Š́dák correction for multiple comparisons. For behavioral studies, all data were screened for fit of distributions with assumptions underlying univariate analyses, which included the Shapiro-Wilk test and q-q plot investigations for normality. Means and standard errors were computed for each measure. Repeated measures analysis of variance (ANOVA) and independent samples $t$-tests were used to analyze behavioral data. Statistical results were confirmed with two-tailed nonparametric testing, when available, for any data sets with violations of the univariate assumptions. Probability value for all analyses was $p<0.05$, unless otherwise stated.

\section{Data availability}

All RNAseq and single-cell RNAseq data have been deposited on the NCBI GEO server with the accession \#GSE165552. All other data associated with this study are presented in the main text or supplementary materials.

Supporting information-This article contains supporting information.

Acknowledgments-We thank Drs. Philip L. Custer and Steven M. Couch for surgical collection of enucleation samples. We thank the Alvin J. Siteman Cancer Center at Washington University School of Medicine and Barnes-Jewish Hospital in St Louis, MO., for the use of the Siteman Flow Cytometry Core. The Siteman Cancer Center is supported in part by an NCI Cancer Center Support Grant \#P30 CA091842. We thank the Genome Technology Access Center in the Department of Genetics at Washington University School of Medicine for help with genomic analyses. The Center is partially supported by the Siteman Cancer Center and by ICTS/CTSA (UL1RR024992) from the National Center for Research Resources (NCRR), a component of the National Institutes of Health (NIH), and NIH Roadmap for Medical Research.

Author contributions-M. D. O. performed the experiments, analyzed data, wrote the first draft, and revised the final draft; C. M. M., K. K. M., J. C., and A. J. D. performed the experiments; L. H. -A., K. D. P., and P. K. R. provided the tumor biopsy material; K. N. W. provided the instruction for mouse xenograft experiments; C. M. Y. performed and analyzed the mouse behavior experiments; P. O. -O. obtained funding, and supervised and analyzed blood pressure and heart rate experiments; J. A. C. obtained funding, analyzed data, and wrote the initial draft; K. J. B. conceived and directed the study, obtained funding, analyzed data, wrote the initial draft, and revised the final draft.

Funding and additional information-This work was supported by NIH grants GM124093 and CA234533 to K. J. B. GM118171 to J. A. C., and HL139754 to P. O. and by an AHA grant 16SDG2726027 to P. O.

Conflict of interest-K. J. B. and M. D. O. are listed as coinventors on a provisional patent application on Targeted Pharmacological Therapeutics In Uveal Melanoma that is owned by Washington University in St. Louis. All other authors declare no competing interests.

Abbreviations-The abbreviations used are: ANOVA, analysis of variance; $\mathrm{DBP}$, diastolic blood pressure; $\mathrm{HR}$, heart rate; $\mathrm{MBP}$, mean blood pressure; PCR2, polycomb repressive complex 2; PDX, patient-derived xenograft; UM, uveal melanoma; SBP, systolic blood pressure.

\footnotetext{
References

1. Van Raamsdonk, C. D., Bezrookove, V., Green, G., Bauer, J., Gaugler, L., O’Brien, J. M., Simpson, E. M., Barsh, G. S., and Bastian, B. C. (2009)
} 
Frequent somatic mutations of GNAQ in uveal melanoma and blue naevi. Nature 457, 599-602

2. Van Raamsdonk, C. D., Griewank, K. G., Crosby, M. B., Garrido, M. C., Vemula, S., Wiesner, T., Obenauf, A. C., Wackernagel, W., Green, G., Bouvier, N., Sozen, M. M., Baimukanova, G., Roy, R., Heguy, A., Dolgalev, I., et al. (2010) Mutations in GNA11 in uveal melanoma. N. Engl. J. Med. 363, 2191-2199

3. Onken, M. D., Worley, L. A., Long, M. D., Duan, S., Council, M. L., Bowcock, A. M., and Harbour, J. W. (2008) Oncogenic mutations in GNAQ occur early in uveal melanoma. Investig. Ophthalmol. Vis. Sci. 49, 5230-5234

4. Küsters-Vandevelde, H. V. N., Klaasen, A., Küsters, B., Groenen, P. J. T. A., Van Engen-Van Grunsven, I. A. C. H., Van Dijk, M. R. C. F., Reifenberger, G., Wesseling, P., and Blokx, W. A. M. (2010) Activating mutations of the GNAQ gene: a frequent event in primary melanocytic neoplasms of the central nervous system. Acta Neuropathol. 119, 317323

5. Sheng, X., Kong, Y., Li, Y., Zhang, Q., Si, L., Cui, C., Chi, Z., Tang, B., Mao, L., Lian, B., Wang, X., Yan, X., Li, S., Dai, J., and Guo, J. (2016) GNAQ and GNA11 mutations occur in 9.5\% of mucosal melanoma and are associated with poor prognosis. Eur. J. Cancer 65, 156-163

6. Joseph, N. M., Brunt, E. M., Marginean, C., Nalbantoglu, Ilk., Snover, D. C., Thung, S. N., Yeh, M. M., Umetsu, S. E., Ferrell, L. D., and Gill, R. M. (2018) Frequent GNAQ and GNA14 mutations in hepatic small vessel neoplasm. Am. J. Surg. Pathol. 42, 1201-1207

7. Shirley, M. D., Tang, H., Gallione, C. J., Baugher, J. D., Frelin, L. P., Cohen, B., North, P. E., Marchuk, D. A., Comi, A. M., and Pevsner, J. (2013) Sturge-Weber syndrome and port-wine stains caused by somatic mutation in GNAQ. N. Engl. J. Med. 368, 1971-1979

8. Bichsel, C. A., Goss, J., Alomari, M., Alexandrescu, S., Robb, R., Smith, L. E., Hochman, M., Greene, A., and Bischoff, J. (2019) Association of somatic GNAQ mutation with Capillary Malformations in a case of choroidal hemangioma. JAMA Ophthalmol. 137, 91-95

9. Nesbit, M. A., Hannan, F. M., Howles, S. A., Babinsky, V. N., Head, R. A., Cranston, T., Rust, N., Hobbs, M. R., Heath, H., and Thakker, R. V. (2013) Mutations affecting G-protein subunit $\alpha 11$ in hypercalcemia and hypocalcemia. N. Engl. J. Med. 368, 2476-2486

10. Li, D., Opas, E. E., Tuluc, F., Metzger, D. L., Hou, C., Hakonarson, H., and Levine, M. A. (2014) Autosomal dominant hypoparathyroidism caused by germline mutation in GNA11: Phenotypic and molecular characterization. J. Clin. Endocrinol. Metab. 99, E1774-E1783

11. Francis, J. H., Milman, T., Grossniklaus, H., Albert, D., Folberg, R., Levitin, G., Coupland, S., Catalanotti, F., Rabady, D., Kandoth, C., Busam, K., and Abramson, D. (2019) GNAQ mutations in Diffuse and Solitary choroidal hemangiomas. Ophthalmology 126, 759-763

12. Krantz, B. A., Dave, N., Komatsubara, K. M., Marr, B. P., and Carvajal, R. D. (2017) Uveal melanoma: epidemiology, etiology, and treatment of primary disease. Clin. Ophthalmol. 11, 279-289

13. Carvajal, R. D., Schwartz, G. K., Tezel, T., Marr, B., Francis, J. H., and Nathan, P. D. (2017) Metastatic disease from uveal melanoma: treatment options and future prospects. Br. J. Ophthalmol. 101, 38-44

14. Kostenis, E., Pfeil, E. M., and Annala, S. (2020) Heterotrimeric Gq proteins as therapeutic targets? J. Biol. Chem. 295, 5206-5215

15. Fujioka, M., Koda, S., Morimoto, Y., and Biemann, K. (1988) Structure of FR900359, a cyclic depsipeptide from Ardisia crenata Sims. J. Org. Chem. $53,2820-2825$

16. Schrage, R., Schmitz, A. L., Gaffal, E., Annala, S., Kehraus, S., Wenzel, D., Büllesbach, K. M., Bald, T., Inoue, A., Shinjo, Y., Galandrin, S., Shridhar, N., Hesse, M., Grundmann, M., Merten, N., et al. (2015) The experimental power of FR900359 to study Gq-regulated biological processes. Nat. Commun. 6, 10156

17. Onken, M. D., Makepeace, C. M., Kaltenbronn, K. M., Kanai, S. M., Todd, T. D., Wang, S., Broekelmann, T. J., Rao, P. K., Cooper, J. A., and Blumer, K. J. (2018) Targeting nucleotide exchange to inhibit constitutively active $G$ protein a subunits in cancer cells. Sci. Signal. 11, eaao6852

18. Lapadula, D., Farias, E., Randolph, C. E., Purwin, T. J., McGrath, D., Charpentier, T. H., Zhang, L., Wu, S., Terai, M., Sato, T., Tall, G. G., Zhou, N., Wedegaertner, P. B., Aplin, A. E., Aguirre-Ghiso, J., et al. (2019)

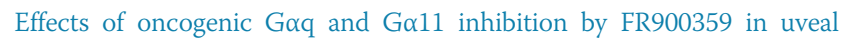
melanoma. Mol. Cancer Res. 17, 963-973

19. Annala, S., Feng, X., Shridhar, N., Eryilmaz, F., Patt, J., Yang, J. H., Pfeil, E. M., Cervantes-Villagrana, R. D., Inoue, A., Häberlein, F., Slodczyk, T., Reher, R., Kehraus, S., Monteleone, S., Schrage, R., et al. (2019) Direct

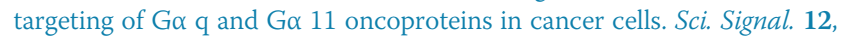
eaau5948

20. Feng, X., Arang, N., Rigiracciolo, D. C., Lee, J. S., Yeerna, H., Wang, Z., Lubrano, S., Kishore, A., Pachter, J. A., König, G. M., Maggiolini, M., Kostenis, E., Schlaepfer, D. D., Tamayo, P., Chen, Q., et al. (2019) A platform of Synthetic lethal gene interaction networks reveals that the GNAQ uveal melanoma oncogene controls the Hippo pathway through FAK. Cancer Cell 35, 457-472.e5

21. Takasaki, J., Saito, T., Taniguchi, M., Kawasaki, T., Moritani, Y., Hayashi, K., and Kobori, M. (2004) A novel Galphaq/11-selective inhibitor. J. Biol. Chem. 279, 47438-47445

22. Kawasaki, T., Taniguchi, M., Moritani, Y., Uemura, T., Shigenaga, T., Takamatsu, H., Hayashi, K., Takasaki, J., Saito, T., and Nagai, K. (2005) Pharmacological properties of YM-254890, a specific G $\alpha$ q/11 inhibitor, on thrombosis and neointima formation in mice. Thromb. Haemost. 94, 184-192

23. Matthey, M., Roberts, R., Seidinger, A., Simon, A., Schröder, R., Kuschak, M., Annala, S., König, G. M., Müller, C. E., Hall, I. P., Kostenis, E., Fleischmann, B. K., and Wenzel, D. (2017) Targeted inhibition of Gq signaling induces airway relaxation in mouse models of asthma. Sci. Transl. Med. 9, eaag2288

24. Meleka, M. M., Edwards, A. J., Xia, J., Dahlen, S. A., Mohanty, I., Medcalf, M., Aggarwal, S., Moeller, K. D., Mortensen, O. V., and Osei-Owusu, P. (2019) Anti-hypertensive mechanisms of cyclic depsipeptide inhibitor ligands for G q/11 class G proteins. Pharmacol. Res. 141, 264-275

25. Sivaraj, K. K., Li, R., Albarran-Juarez, J., Wang, S., Tischner, D., Grimm, M., Swiercz, J. M., Offermanns, S., and Wettschureck, N. (2015) Endothelial $G \alpha_{\mathrm{q} / 11}$ is required for VEGF-induced vascular permeability and angiogenesis. Cardiovasc. Res. 108, 171-180

26. Dettlaff-Swiercz, D. A., Wettschureck, N., Moers, A., Huber, K., and Offermanns, S. (2005) Characteristic defects in neural crest cell-specific

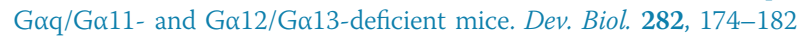

27. Graham, D. L., Buendia, M. A., Chapman, M. A., Durai, H. H., and Stanwood, G. D. (2015) Deletion of Goq in the telencephalon alters specific neurobehavioral outcomes. Synapse 69, 434-445

28. Wettschureck, N., Rütten, H., Zywietz, A., Gehring, D., Wilkie, T. M., Chen, J., Chien, K. R., and Offermanns, S. (2001) Absence of pressure overload induced myocardial hypertrophy after conditional inactivation of Goq/Ga11 in cardiomyocytes. Nat. Med. 7, 1236-1240

29. Offermanns, S., Zhao, L. P., Gohla, A., Sarosi, I., Simon, M. I., and Wilkie, T. M. (1998) Embryonic cardiomyocyte hypoplasia and craniofacial de-

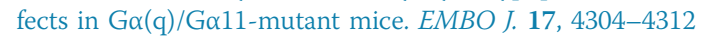

30. Hitchman, T. D., Bayshtok, G., Ceraudo, E., Moore, A. R., Lee, C., Jia, R., Wang, N., Pachai, M. R., Shoushtari, A. N., Francis, J. H., Guan, Y., Chen, J., Chang, M. T., Taylor, B. S., Sakmar, T. P., et al. (2020) Combined inhibition of Goq and MEK Enhances therapeutic efficacy in uveal melanoma. Clin. Cancer Res. https://doi.org/10.1158/1078-0432.ccr-20-2860

31. Johnson, C. P., Kim, I. K., Esmaeli, B., Amin-Mansour, A., Treacy, D. J., Carter, S. L., Hodis, E., Wagle, N., Seepo, S., Yu, X., Lane, A. M., Gragoudas, E. S., Vazquez, F., Nickerson, E., Cibulskis, K., et al. (2017) Systematic genomic and translational efficiency studies of uveal melanoma. PLoS One 12, e0178189

32. Robertson, A. G., Shih, J., Yau, C., Gibb, E. A., Oba, J., Mungall, K. L., Hess, J. M., Uzunangelov, V., Walter, V., Danilova, L., Lichtenberg, T. M., Kucherlapati, M., Kimes, P. K., Tang, M., Penson, A., et al. (2017) Integrative analysis identifies four molecular and clinical Subsets in uveal melanoma. Cancer Cell 32, 204-220.e15

33. Onken, M. D., Worley, L. A., Ehlers, J. P., and Harbour, J. W. (2004) Gene expression profiling in uveal melanoma reveals two molecular classes and predicts metastatic death. Cancer Res. 64, 7205-7209

34. Onken, M. D., Worley, L. A., Char, D. H., Augsburger, J. J., Correa, Z. M., Nudleman, E., Aaberg, T. M., Altaweel, M. M., Bardenstein, D. S., Finger, P. T., Gallie, B. L., Harocopos, G. J., Hovland, P. G., McGowan, H. D., 


\section{EDITORS' PICK: FR900359 targets primary and metastatic uveal melanoma}

Milman, T., et al. (2012) Collaborative ocular oncology group report number 1: prospective validation of a multi-gene prognostic assay in uveal melanoma. Ophthalmology 119, 1596-1603

35. Harbour, J. W., Onken, M. D., Roberson, E. D. O., Duan, S., Cao, L., Worley, L. A., Council, M. L., Matatall, K. A., Helms, C., and Bowcock, A. M. (2010) Frequent mutation of BAP1 in metastasizing uveal melanomas. Science (80-. ) 330, 1410-1413

36. Griewank, K. G., Yu, X., Khalili, J., Sozen, M. M., Stempke-Hale, K., Bernatchez, C., Wardell, S., Bastian, B. C., and Woodman, S. E. (2012) Genetic and molecular characterization of uveal melanoma cell lines. Pigment Cell Melanoma Res. 25, 182-187

37. Amirouchene-Angelozzi, N., Nemati, F., Gentien, D., Nicolas, A., Dumont, A., Carita, G., Camonis, J., Desjardins, L., Cassoux, N., PipernoNeumann, S., Mariani, P., Sastre, X., Decaudin, D., and Roman-Roman, S. (2014) Establishment of novel cell lines recapitulating the genetic landscape of uveal melanoma and preclinical validation of mTOR as a therapeutic target. Mol. Oncol. 8, 1508-1520

38. Brantley, J., and Harbour, J. W. (2000) Deregulation of the Rb and p53 pathways in uveal melanoma. Am. J. Pathol. 157, 1795-1801

39. Kawasaki, T., Taniguchi, M., Moritani, Y., Hayashi, K., Saito, T., Takasaki, J., Nagai, K., Inagaki, O., and Shikama, H. (2003) Antithrombotic and thrombolytic efficacy of YM-254890, a Gq/II inhibitor, in a rat model of arterial thrombosis. Thromb. Haemost. 90, 406-413

40. Wettschureck, N., and Offermanns, S. (2005) Mammalian G proteins and their cell type specific functions. Physiol. Rev. 85, 1159-1204.

41. Chen, X., Wu, Q., Depeille, P., Chen, P., Thornton, S., Kalirai, H., Coupland, S. E., Roose, J. P., and Bastian, B. C. (2017) RasGRP3 Mediates MAPK pathway activation in GNAQ mutant uveal melanoma. Cancer Cell 31, 685-696.e6

42. Chua, V., Lapadula, D., Randolph, C., Benovic, J. L., Wedegaertner, P., and Aplin, A. E. (2017) Dysregulated GPCR signaling and therapeutic options in uveal melanoma. Mol. Cancer Res. 15, 501-506

43. Wöll, E., Bedikian, A., and Legha, S. S. (1999) Uveal melanoma: Natural history and treatment options for metastatic disease. Melanoma Res. 9, $575-581$
44. Gonsalves, C. F., Eschelman, D. J., Adamo, R. D., Anne, P. R., Orloff, M. M., Terai, M., Hage, A. N., Yi, M., Chervoneva, I., and Sato, T. (2019) A prospective Phase II trial of radioembolization for treatment of uveal melanoma hepatic metastasis. Radiology 293, 223-231

45. Eschelman, D. J., Gonsalves, C. F., and Sato, T. (2013) Transhepatic therapies for metastatic uveal melanoma. Semin. Intervent. Radiol. 30, $39-48$

46. Singh, A. D., Kalyani, P., and Topham, A. (2005) Estimating the risk of malignant transformation of a choroidal nevus. Ophthalmology 112, $1784-1789$

47. Evelyn, C. R., Wade, S. M., Wang, Q., Wu, M., Iniguez-Lluhi, J. A., Merajver, S. D., and Neubig, R. R. (2007) CCG-1423: a small-molecule inhibitor of RhoA transcriptional signaling. Mol. Cancer Ther. 6, 2249-2260

48. Delston, R. B., Matatall, K. A., Sun, Y., Onken, M. D., and Harbour, J. W. (2011) P38 phosphorylates Rb on Ser567 by a novel, cell cycleindependent mechanism that triggers $\mathrm{Rb}-\mathrm{Hdm} 2$ interaction and apoptosis. Oncogene 30, 588-599

49. Matatall, K. A., Agapova, O. A., Onken, M. D., Worley, L. A., Bowcock, A. M., and Harbour, J. W. (2013) BAP1 deficiency causes loss of melanocytic cell identity in uveal melanoma. BMC Cancer 13, 371

50. Subramanian, A., Tamayo, P., Mootha, V. K., Mukherjee, S., Ebert, B. L., Gillette, M. A., Paulovich, A., Pomeroy, S. L., Golub, T. R., Lander, E. S., and Mesirov, J. P. (2005) Gene set enrichment analysis: a knowledgebased approach for interpreting genome-wide expression profiles. Proc. Natl. Acad. Sci. U. S. A. 102, 15545-15550

51. Owens, E. A., Jie, L., Reyes, B. A. S., Van Bockstaele, E. J., and OseiOwusu, P. (2017) Elastin insufficiency causes hypertension, structural defects and abnormal remodeling of renal vascular signaling. Kidney Int. 92, 1100-1118

52. Wozniak, D. F., Hartman, R. E., Boyle, M. P., Vogt, S. K., Brooks, A. R., Tenkova, T., Young, C., Olney, J. W., and Muglia, L. J. (2004) Apoptotic neurodegeneration induced by ethanol in neonatal mice is associated with profound learning/memory deficits in juveniles followed by progressive functional recovery in adults. Neurobiol. Dis. 17, $403-414$ 